\title{
VviERF6Ls: an expanded clade in Vitis responds transcriptionally to abiotic and biotic stresses and berry development
}

\author{
Haley S. Toups ${ }^{1}$, Noé Cochetel ${ }^{1}$, Dennis Gray ${ }^{2}$ and Grant R. Cramer ${ }^{1 *}$ (D)
}

\begin{abstract}
Background: VviERF6Ls are an uncharacterized gene clade in Vitis with only distant Arabidopsis orthologs. Preliminary data indicated these transcription factors may play a role in berry development and extreme abiotic stress responses. To better understand this highly duplicated, conserved clade, additional members of the clade were identified in four Vitis genotypes. A meta-data analysis was performed on publicly available microarray and RNA-Seq data (confirmed and expanded with RT-qPCR), and Vitis VviERF6L1 overexpression lines were established and characterized with phenotyping and RNA-Seq.

Results: A total of 18 PN40024 VviERF6Ls were identified; additional VviERF6Ls were identified in Cabernet Sauvignon, Chardonnay, and Carménère. The amino acid sequences of VviERF6Ls were found to be highly conserved. VviERF6L transcripts were detected in numerous plant organs and were differentially expressed in response to numerous abiotic stresses including water deficit, salinity, and cold as well as biotic stresses such as red blotch virus, N. parvum, and E. necator. VviERF6Ls were differentially expressed across stages of berry development, peaking in the pre-veraison/veraison stage and retaining conserved expression patterns across different vineyards, years, and Vitis cultivars. Co-expression network analysis identified a scarecrow-like transcription factor and a calmodulin-like gene with highly similar expression profiles to the VViERF6L clade. Overexpression of VviERF6L1 in a Seyval Blanc background did not result in detectable morphological phenotypes. Genes differentially expressed in response to VViERF6L1 overexpression were associated with abiotic and biotic stress responses.
\end{abstract}

Conclusions: VviERF6Ls represent a large and distinct clade of ERF transcription factors in grapevine. The high conservation of protein sequence between these 18 transcription factors may indicate these genes originate from a duplication event in Vitis. Despite high sequence similarity and similar expression patterns, VviERF6Ls demonstrate unique levels of expression supported by similar but heterogeneous promoter sequences. VViERF6L gene expression differed between Vitis species, cultivars and organs including roots, leaves and berries. These genes respond to berry development and abiotic and biotic stresses. VViERF6L1 overexpression in Vitis vinifera results in differential expression of genes related to phytohormone and immune system signaling. Further investigation of this interesting gene family is warranted.

Keywords: ERF6L, Ethylene response factor, Motif, Vitis vinifera

\footnotetext{
*Correspondence: cramer@unr.edu

${ }^{1}$ Department of Biochemistry and Molecular Biology, University of Nevada, Reno, NV 89557, USA

Full list of author information is available at the end of the article
}

\section{$\triangle B M C$}

(c) The Author(s). 2020 Open Access This article is licensed under a Creative Commons Attribution 4.0 International License, which permits use, sharing, adaptation, distribution and reproduction in any medium or format, as long as you give appropriate credit to the original author(s) and the source, provide a link to the Creative Commons licence, and indicate if changes were made. The images or other third party material in this article are included in the article's Creative Commons licence, unless indicated otherwise in a credit line to the material. If material is not included in the article's Creative Commons licence and your intended use is not permitted by statutory regulation or exceeds the permitted use, you will need to obtain permission directly from the copyright holder. To view a copy of this licence, visit http://creativecommons.org/licenses/by/4.0/ The Creative Commons Public Domain Dedication waiver (http://creativecommons.org/publicdomain/zero/1.0/) applies to the data made available in this article, unless otherwise stated in a credit line to the data. 


\section{Background}

Ethylene is a key phytohormone with roles in plant growth and development [1] as well as abiotic and biotic stress responses [2-4]. Vitis vinifera (grapevine) is a non-climacteric fruit that does not ripen with a respiratory burst of ethylene, instead maturing with increased abscisic acid (ABA) concentration. However, ethylene plays an important role in fruit development as berries transition into veraison, the beginning stage of color development and berry softening, initiating ethylene signaling and activating Ethylene Response Factors (ERFs) [5-7]. ERFs regulate gene expression of targets including transcription factors like RESPONSIVE TO DEHYDRATION 29B (RD29B), LATE EMBRYOGENESIS 4-5 (LEA4-5), HEAT SHOCK PROTEIN 101 (HSP101), and other $E R F S$, resulting in physiological responses and adaptations that allow a plant to better survive under specific environmental conditions like water deficit and high temperature [8]. These transcription factors act as major signaling hubs that integrate cross-talk between ethylene and other phytohormones to mediate gene expression [9-14]. ERFs belong to the APETLA2/ERF Family consisting of over 122 and 149 genes in Arabidopsis [15] and Vitis [4], respectively. This family is divided into 12 sub-families based on regulatory elements and DNA-binding domains.

Previously, a unique Vitis clade was identified in subfamily IX, consisting of 12 members with no Arabidopsis ortholog [16]. Sequence analysis revealed these genes most closely resembled AtERF6. This clade was named ERF6-like (ERF6L) after the closest Arabidopsis ortholog, and the genes were numbered from one through 12 based on chromosomal coordinates of the V1 structural annotation V2 assembly of PN40024 [16]. Affymetrix and NimbleGen grapevine microarrays with limited probe sets hybridizing to some of the $V v i E R F 6 L$ genes revealed $V$ viERF6Ls were differentially expressed in berry skins across berry ripening [16] and in leaves in response to severe leaf dehydration [17].

Recently, a new improved structural annotation (V3) of the PN40024 genome (V2 assembly) was released, providing additional gene loci [18]. In this report, the early observations of the VviERF6L clade are investigated further. These genes were analyzed using the new structural annotation of PN40024 [18]; to better understand the role of this clade in Vitis, gene expression patterns were queried in a meta-data analysis and novel experimental treatments were performed. VviERF6L1 overexpression lines were established and phenotyped. Manual curation of the new structural annotations resulted in the discovery of additional VviERF6Ls not previously identified. VviERF6L expression was dependent on cultivar, species, organ, hormone and stress treatments.

\section{Results}

The VviERF6L clade was expanded to 18 members in the PN40024 reference genome

Novel VviERF6Ls genes were discovered by manually searching for conserved amino acid (AA) motifs in the newly annotated PN40024 genome. Along with the 12 previously identified $V v i E R F 6 L$ genes [16], five additional genes were first identified (Table 1) from this manual curation. These additional genes were identified in unannotated sections of chromosome sequences by searching for specific AA motifs across the individual chromosomes using tools in ORCAE [19] where the reference grape genome sequence, PN40024, is stored. Structural models were confirmed in ORCAE using both mRNA [20] and expressed sequence tag (EST) data to confirm $5 '$ and $3^{\prime}$ ends of annotated sequences [21].

An additional VviERF6L was identified with an insilico detection strategy. The manually curated VviERF6Ls were confirmed and substantiated as members of this clade from protein motifs identified in MEME [22, 23]. MEME revealed VviERF6L proteins consist of nine highly conserved AA motifs (Fig. 1 and Additional Files 1 and 2). The nine motifs are referred to in order of E-value with the lowest value motif corresponding to Motif 1 (Additional File 2). To identify additional novel VviERF6Ls that may have been overlooked with the manual annotation, an in-silico detection strategy was devised using the first (Motif 5) and last (Motif 4) spatial AA motifs to query the Vitis proteome. Genome coordinates that contained either the first, last, or both motifs were extracted corresponding to the potential proteins containing the motif(s) of interest. When only the first or the last motif was detected, the putative protein sequence was extended to 280 AA to obtain the potential full-length protein. This strategy confirmed the five novel VviERF6L genes from the manual curation and identified a sixth, increasing the members of the VviERF6L clade from 12 in the V1 annotation to 18 in the V2 annotation of PN40024 (Table 1).

The nine VviERF6L protein motifs were detected as being significantly present $\left(p<1.73 \times 10^{-179}\right)$ and conserved $\left(\mathrm{E}<8.8 \times 10^{-14}\right)$ among the 18 VviERF6Ls (Additional File 2). These motifs had an average length of $30 \mathrm{AA}$ with the longest and shortest motifs (Motif 1 and Motif 9) having lengths of 50 and 7 AA, respectively (Additional File 2), resultant of the MEME settings used. Specific VviERF6L protein motif sequence and location per VviERF6L can be found in Additional File 3. Four protein motifs were identifiable and had previously been characterized as regulatory domains of other ERF Group IX TFs (Fig. 1) [15]. These protein motifs included the AP2/ERF domain (DNA-binding; Motifs 1 and 2), the CMIX-2 (N-terminal acidic transactivation; Motif 5) the CMIX-5 (MAP kinase phosphorylation site; Motif 4), 
Table 1 PN40024 VviERF6L gene names and coordinates. Common gene names, V2 and V3 PN40024 loci annotation names, start and stop gene chromosome coordinates, coding strands, and total protein lengths in amino acid residues of the 18 identified VViERF6Ls in PN40024

PN40024 VVIERF6LS

\begin{tabular}{|c|c|c|c|c|c|c|}
\hline Gene Name & Loci name V3 & Loci name V2 & start & stop & strand & Length $(A A)$ \\
\hline VviERF6L1 & Vitvi16g00350 & VIT_16s0013g00900 & $6,283,579$ & $6,284,605$ & + & 278 \\
\hline VviERF6L2 & Vitvi16g01429 & VIT_16s0013g00950 & $6,323,868$ & $6,324,845$ & + & 278 \\
\hline VviERF6L3 & Vitvi16g01438 & VIT_16s0013g00970 & $6,340,458$ & $6,341,282$ & + & 275 \\
\hline VviERF6L4 & Vitvi16g01430 & VIT_16s0013g00980 & $6,353,674$ & $6,354,603$ & + & 276 \\
\hline VviERF6L5 & Vitvi16g01424 & VIT_16s0013g00990 & $6,374,230$ & $6,375,289$ & + & 278 \\
\hline VviERF6L6 & Vitvi16g01432 & VIT_16s0013g01000 & $6,390,945$ & $6,391,977$ & - & 269 \\
\hline VviERF6L7 & Vitvi16g00362 & VIT_16s0013g01050 & $6,495,312$ & $6,496,325$ & + & 278 \\
\hline VviERF6L8 & Vitvi16g00363 & VIT_16s0013g01060 & $6,518,283$ & $6,519,283$ & + & 278 \\
\hline VviERF6L9 & Vitvi16g00370 & VIT_16s0013g01070 & $6,550,999$ & $6,552,087$ & + & 276 \\
\hline VviERF6L10 & Vitvi16g01437 & VIT_16s0013g01090 & $6,626,070$ & $6,627,309$ & - & 265 \\
\hline VviERF6L11 & Vitvi16g01434 & VIT_16s0013g01110 & $6,659,232$ & $6,660,318$ & - & 279 \\
\hline VviERF6L12 & Vitvi16g00380 & VIT_16s0013g01120 & $6,692,405$ & $6,693,620$ & + & 243 \\
\hline VviERF6L13 & Vitvi16g01423 & NA & $6,387,963$ & $6,388,894$ & + & 275 \\
\hline VviERF6L14 & Vitvi16g00360 & NA & $6,460,898$ & $6,461,806$ & + & 191 \\
\hline VviERF6L15 & Vitvi16g01442 & NA & $6,530,589$ & $6,531,483$ & + & 275 \\
\hline VviERF6L16 & Vitvi16g01444 & NA & $6,621,652$ & $6,622,826$ & - & 283 \\
\hline VviERF6L17 & Vitvi16g01443 & NA & $6,662,810$ & $6,663,750$ & - & 265 \\
\hline VviERF6L18 & Vitvi16g01435 & NA & $6,667,987$ & $6,669,132$ & - & 279 \\
\hline
\end{tabular}

and the CMIX-6 (MAP kinase phosphorylation site; Motif 9) domains (Fig. 1). Motifs 1, 2, and 7 were present in all 18 VviERF6Ls. Motifs 3, 4, 5, 6, and 9 were present in $77.8 \%$ of VviERF6L proteins, and Motif 8 was present in nine of the 18 VviERF6Ls (calculated from Additional File 3).

The VviERF6L AP2/ERF domain is homologous to that of Arabidopsis (At)ERF1 and 096. To identify VviERF6L sequence conservation with proteins in Arabidopsis thaliana, the nine motifs were queried in InterPro and the AP2/ERF domain was modeled in SWISS-model $[24,25]$. The AP2/ERF domain (Motifs 1 and 2) of VviERF6L1, 2, 3, 4, 5, 6, 7, and 13 had the closest identity with that of AtERF1 with an average identity of 75.5\% (Additional File 4). VviERF6L8, 9, 10, 11, 12, 15, 16,17 , and 18 had an average identity of $70.8 \%$ with the AtERF096 AP2/ERF domain, identified as the closest ortholog (Additional File 4).

VviERF6L12 and 14 appear to be truncated proteins. VviERF6L14 lacks the first $4 \mathrm{~N}$-terminal motifs (Fig. 1), with no matching publicly available RNA-Seq or EST reads and insufficient sequence information in this region of the PN40024 genome in ORCAE. Besides VviERF6L15, VviERF6L14 is the only other non-monoexonal VviERF6L. The true start codon of VviERF6L14 may exist in what is currently the un-sequenced region that is presently annotated as an intron and can be viewed in ORCAE [19]. Despite potential mis-annotation of VviERF6L14 gene coordinates, promoter (see later) and protein motif analysis (Fig. 1, and Additional Files 1 and 2) validate this gene as a VviERF6L. VviERF6L12 appears to be a functional truncated protein (Fig. 1), supported by mRNA and EST read mapping across the length of the transcript in ORCAE. VviERF6L12 lacks the first $4 \mathrm{~N}$-terminal motifs, which correspond to potential regulatory domains including the CMIX-2 domain (Fig. 1). VviERF6L12 is also missing Motif 4 corresponding to a CMIX-5 domain. VviERF6L3, 6, 9, 11, 13, 16, and 18 do not share consensus Motif 8 (Fig. 1). These proteins have higher amino acid variability in this region. VviERF6L10, 15, and 17 are also missing Motif 4 (Fig. 1).

The 18 VviERF6L proteins are a conserved clade. A multiple sequence comparison by log-expectation (MUSCLE) multiple sequence alignment (MSA) was performed to better understand the diversity within the VviERF6L clade. Percent identity was extracted from a MUSCLE alignment (Fig. 2). The 18 VviERF6Ls share high sequence conservation (average of $73.8 \%$ ), with VviERF6L12, one of the truncated VviERF6Ls, diverging the most with an average percent identity of $50.9 \%$ (calculated from Fig. 2).

The VviERF6L clade is expanded in Vitis vinifera relative to other plant species. The number of ERF6L 


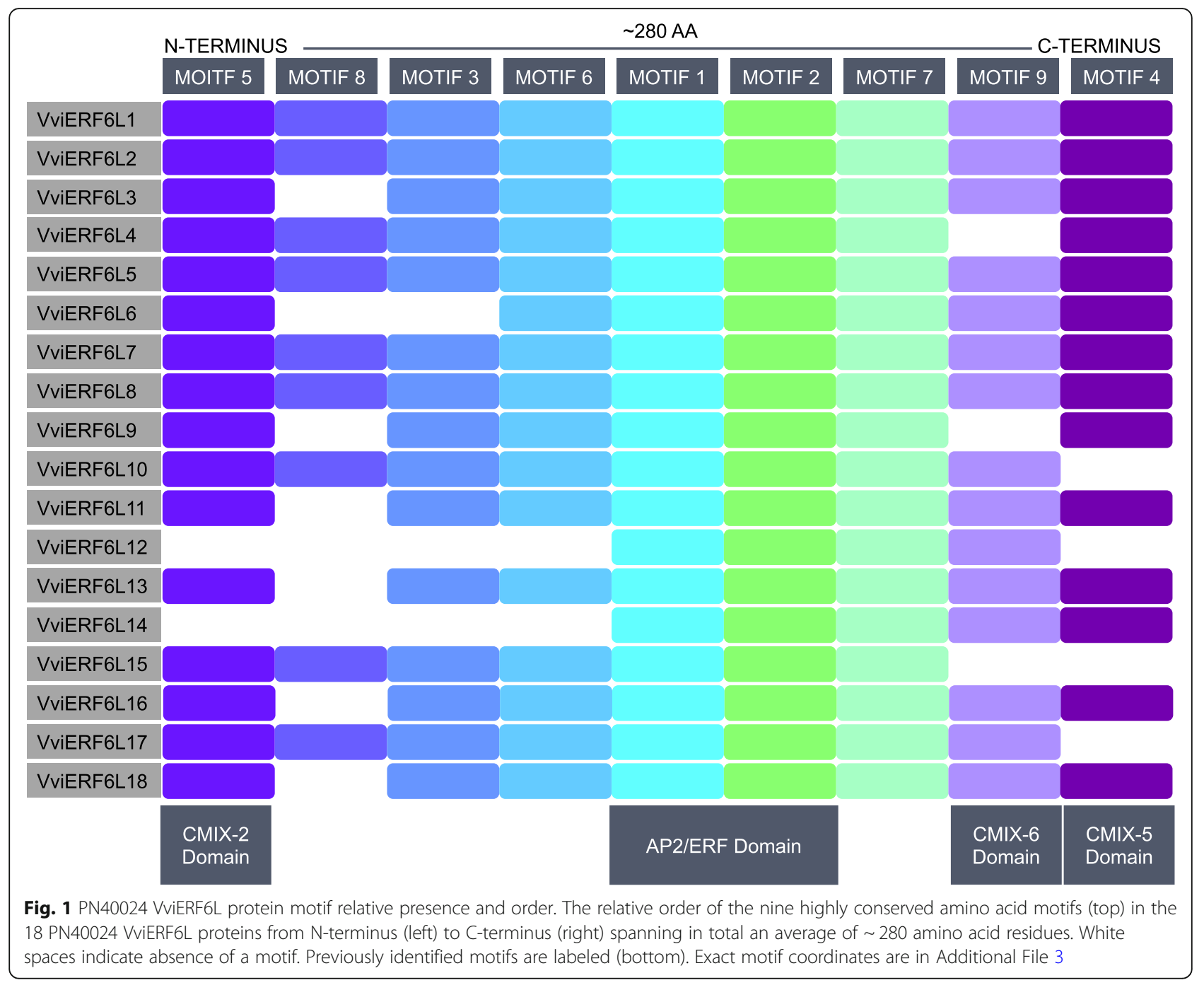

paralogs were identified in the species that had the genes with the highest orthology to VviERF6L1 from the Pantaxonomic Compara Gene Tree in Gramene update 2018 containing 44 genomes [26] including the V1 annotation of PN40024 [20]. The number of potential ERF6L orthologs was quantified in carrot (D. carota), soybean (G. max), tomato (S. lycopersicum), and potato (S. tuberosum) (Additional File 5). Vitis vinifera had 4.5fold more ERF6L paralogs than tomato and potato, 9fold more than soybean, and 17 more potential ERF6L genes than carrot (Additional File 5).

The VviERF6L clade is expanded across Vitis genotypes Additional VviERF6Ls were identified in the translated Cabernet Sauvignon (CS) genome [27] indicating the VviERF6L clade members vary with grape genotypes. The nine PN40024 VviERF6L protein motifs were utilized to detect VviERF6Ls in the proteome sequence of CS using TOMTOM [23]. Translated genes that contained at least three of the nine PN40024 VviERF6L protein motifs were extracted and analyzed with MEME as potential VviERF6Ls. These genes were used to identify CS specific VviERF6L protein motifs. TOMTOM used the CS cultivar specific VviERF6L protein motifs to identify additional potential CS VviERF6Ls that were missed using the nine PN40024 motifs (Additional Files 2 and $6,7,8,9)$. Thirteen highly conserved $\left(\mathrm{E}<1.3 \times 10^{-2}\right)$ CS protein motifs (Additional File 2) were identified. The CS protein motifs were very similar to those of PN40024 (Additional Files 1, 7, 9 and 10). Homology between PN40024 and CS VviERF6L protein motifs was quantified with protein BLAST [28] (Additional File 10). CS Motifs 1-6 shared 100\% identity with corresponding PN40024 motifs, and CS Motif 7 shared $\sim 71 \%$ with PN40024 Motif 9 (Additional File 10). In total, 26 CS VviERF6Ls were identified (Additional Files 8, 9, and 11). Interestingly, unique $V v i E R F 6 L$ sequences were identified in CS like VvCabSauv08_H0036F 008.ver1.0.g139880, which appears to be a novel VviERF6L not conserved in PN40024 (Additional Files 12 


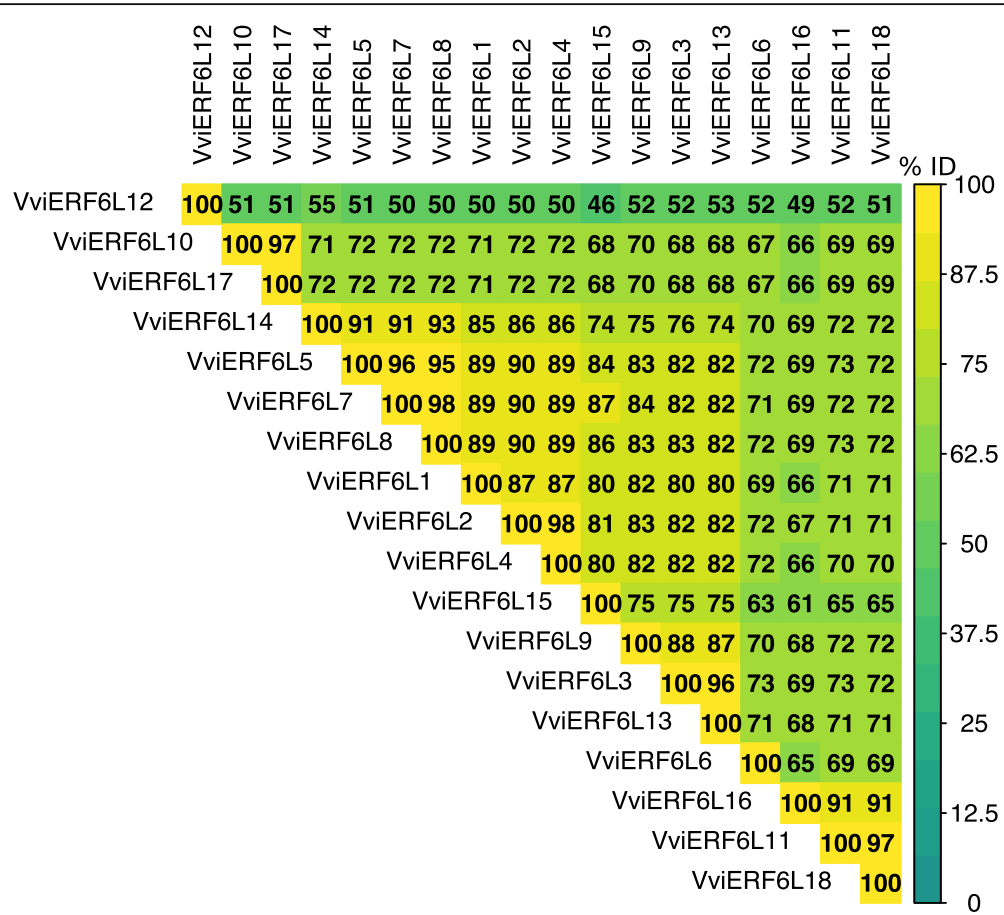

Fig. 2 Percent identity of the 18 PN40024 VviERF6L proteins. Sequences were aligned with a MUSCLE multiple sequence alignment and compared to all other sequences with blue representing lower and yellow representing higher percent identity (\% ID)

and 13). Lengths of CS VviERF6L proteins are in Additional File 11. CS VviERF6Ls ( 300 AA residues) were on average 20 AA residues longer than PN40024 ( 280 AA residues) (Table 1 and Additional File 11). CS lacked paralogs similar to PN40024 VviERF6L3, 8, 11, and 14 and had distinct VviERF6Ls (like VvCabSauv08_ P0367F.ver1.0.g601540 and VvCabSauv08_H0036F_ 008.ver1.0.g139880), without a clearly distinguishable PN40024 equivalent. VvCabSauv08_H0036F_ 008.ver1.0.g139910 (596 AA residues) contained duplicated Motif 1-4, 5-9, and 11 (Additional Files 6 and 9). VvCabSauv08_H0036F_008.ver1.0.g139950 (839 AA residues) consisted of duplicate Motif 1, 2, 7, and 12 and triplicate Motif 3, 5, 6, and 11. These two genes were about two and three times the length of the average CS VviERF6L ( 300 AA residues (calculated from Additional File 10)), respectively. VvCabSauv08_H0036F_ 008.ver1.0.g139990 was a severely truncated VviERF6L (106 AA residues), completely lacking any conserved Nterminal motif (Additional Files 6 and 9). VvCabSauv08 P0070F.ver1.0.g450750 was of comparable length (243 AA residues) to the average CS VviERF6L, but this gene had more variable sequence, containing only four of the thirteen conserved motifs (Additional Files 6 and 9).

Chardonnay $(\mathrm{CH})$ [29] and Carménère (CA) [30] also have expanded VviERF6L clades with 15 and 14 VviERF6Ls respectively (Additional Files 12 and 13). VviERF6Ls from PN40024 and CS were queried in protein BLAST in genome sequences of $\mathrm{CH}$ and $\mathrm{CA}$ to identify VviERF6Ls in these genotypes. The genomes of $\mathrm{CH}$ and CA were not released at this time; only BLAST was publicly available. Additional novel VviERF6Ls may exist in these genotypes, which may be identified using the motif detection strategy described for PN40024 and CS when the genomes become fully available.

The PN40024, CS, CH, and CA VviERF6Ls were more similar across Vitis vinifera genotypes than to other VviERF proteins (Additional File 12). To distinguish relationships between the highly homologous members of the VviERF6L clade in the AP2/ERF subfamily IX [16], a maximum likelihood phylogenetic tree was generated from Vitis vinifera PN40024, CS, CH, and CA VviERF6L paralogs and PN40024 VviERF proteins (gene names and protein sequences available in Additional File 13). The tree was created using the Jones-Taylor-Thornton model with the Bootstrap method test in MEGA X [31]. Sequences were extracted from the PN40024 V2 assembly V3 structural annotation [18], CS genome [27], $\mathrm{CH}$ BLAST-tool [29], and the CA BLAST tool [30]. All predicted VviERF6L proteins grouped together from the four genotypes examined (Additional File 12). Vitvi05g01525, corresponding to a putative disease related PRF protein [21], clustered with $\mathrm{CH}$ and CA VviERF6Ls in the multi-gentoype VviERF6L clade. This gene is inadequately sequenced on ORCAE, having 4512 N's in the coding sequence, and it is unclear if this gene is 
correctly positioned or annotated. The VviERF6L clade is distinct from other members of the AP2/ERF family (Additional File 12), and the VviERF6L protein sequences are highly conserved.

\section{VviERF6L promoter regions are distinct with several conserved motifs}

The PN40224 VviERF6Ls have variable promoter regions with several conserved and repeated cis-acting elements. To gain insight into the transcriptional regulation of the highly conserved VviERF6L genes in the PN40024 genome, $-3000 \mathrm{bp}$ upstream from the transcription start site (TSS) for the 18 PN40024 VviERF6Ls was analyzed with PLACE [32], and a multiple sequence alignment was performed to compare the putative promoter regions (Fig. 3). These regions showed greater diversity than VviERF6L protein sequences, averaging 48.7\% relative to $81.05 \%$ identity (calculated from DNA sequences). PN40024 VviERF6L promoter region motif coordinates are in Additional File 14. A total of 200 known motifs were identified in the VviERF6L - $3000 \mathrm{bp}$ putative promoter regions (calculated from Additional File 14). Of these cis-elements, 42 were present in all VviERF6L upstream regions (Additional File 15). The CAATBOX1 was the most represented motif across VviERF6L putative promoters, repeated 885 times, followed by DOFCOREZM (864 repetitions) and CACTFTPPCA1 (845 repetitions) (Additional File 15). These three motifs had an average of $\sim 46$ repeats per VviERF6L promoter. Numerous other cis-elements were repeated hundreds of times including ARR1AT and MYCCONSENSUSAT motifs.

Although the VviERF6Ls shared several highly repeated cis-regulatory elements, there were numerous differences across the VviERF6L promoter regions. The VviERF6L12 promoter region contained the highest number of ROOTMOTIFTAPOX1 (82 repeats), with the closest VviERF6L, VviERF6L14 having 41 repeats and the other VviERF6Ls having even fewer. The VviERF6L12 promoter region also had the most duplication of TATABOX3, ACGTATERD1 (analogous with VviERF6L1), SEF1MOTIF, SEF4MOTIFGM7S, WBOXATNPR1, and LECPLEACS2 (Additional Files 15 and 16). Three unique motifs were detected in the promoter region of VviERF6L12 that were not present in any other $V v i E R F 6 L$ promoter: ABREZMRAB28, PALBOXLPC and UP1ATMASD (Additional File 14). Although the VviERF6L protein sequences are highly conserved, there is considerable variation in the VviERF6L promoter regions, indicating these genes are under unique transcriptional regulation.

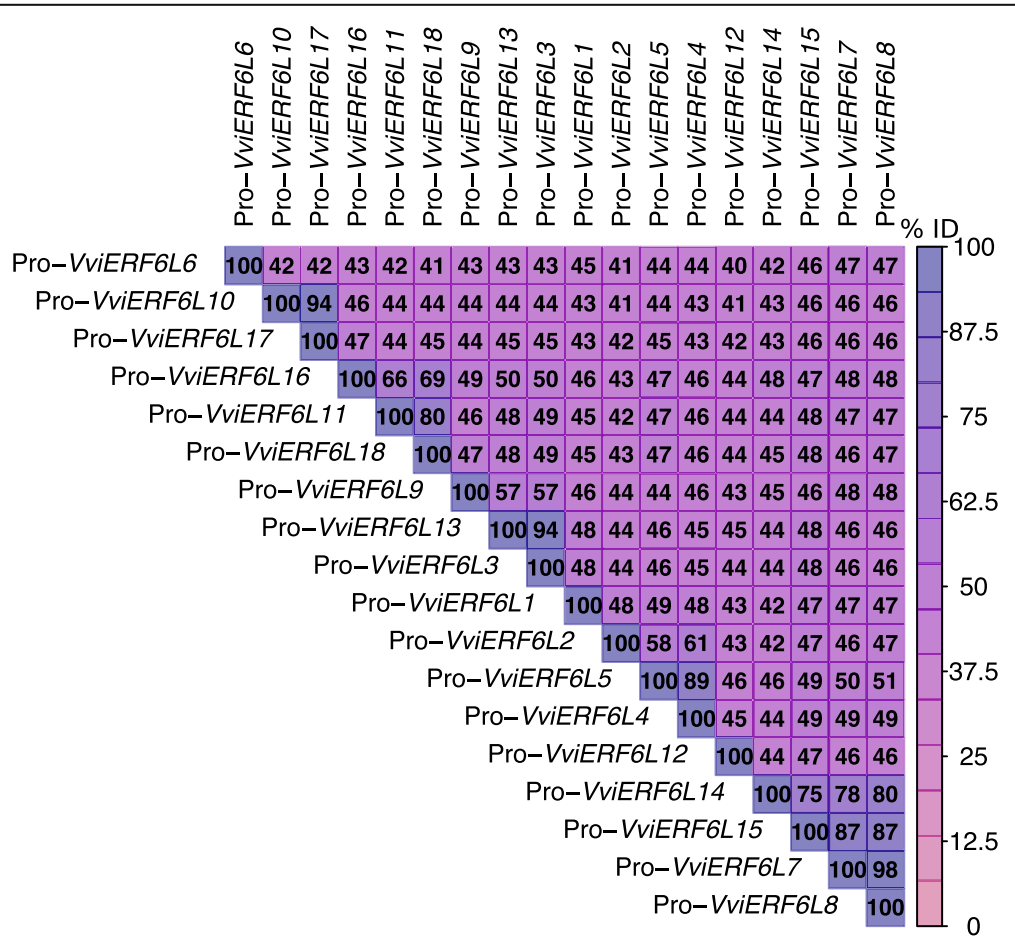

Fig. 3 Percent identity of the 18 PN40024 VviERF6L putative promoter regions (- 3000 bp). Promoter (Pro-) sequences ( -3000 bp) were aligned with a MUSCLE multiple sequence alignment and compared to all other sequences with pink representing lower and purple representing higher percent identity (\% ID) 
VviERF6L genes are expressed in many organs and tissues Examining the grapevine gene atlas [33], VviERF6Ls were expressed in numerous grapevine organs, across developmental stages, and in tissues including berries, stamen, buds, tendrils, flowers, pollen, seeds, leaves, and roots (Additional File 17). VviERF6L1, 5, and 12 were the most commonly expressed $V v i E R F 6 L s$ across various tissues. VviERF6L6, 10, and 11 were less broadly expressed across tissues. VviERF6L6 was only expressed in the rachis, carpel, petal, leaves, roots, and buds while VviERF6L10 and 11 were expressed in these tissues as well as berry flesh. VviERF6L8 was expressed in the same number of tissues as VviERF6L2 and 3 and comparable to VviERF6L4 and 7. Breaking down the berry into pulp, seed, and skin VviERF6Ls generally had significantly higher expression in the skin at pre-veraison and seed at maturity when the berries are red, soft, and ready to harvest (RSH) (Additional File 18) [GSE49569] [34]. VviERF6L12 was the only VviERF6L to increase in signal intensity in the pulp as berries developed, and this gene maintained the highest expression level in all berry tissues at all developmental stages.

\section{VviERF6L meta-data analysis parameters}

VviERF6L expression was extensively examined across existing transcriptomic data in the literature. To better differentiate and understand the potential functional roles of VviERF6L genes in Vitis, VviEFRF6L gene expression was examined in a meta-data analysis of VviERF6L gene expression performed using 75 publicly available microarray and 24 RNA-Seq data series downloaded from NCBI Gene Expression Omnibus (GEO) [35] and Sequence Read Archive [36] data bases. The following data are examples of results found, but many other datasets demonstrate similar patterns. The example data series selected are simplified for visualization purposes. The data series (Additional File 19) investigated for $V v i E R F 6 L$ gene expression met the following criteria: the experiment contained at least three individual biological or experimental replicates, $V v i E R F 6 L$ gene expression was detectable, and at least one VviERF6L was identified as a differentially expressed gene (DEG) in the author's original differential expression analysis (DEA). Results are discussed based on the author's original DEA and statistical analysis unless otherwise specified.

Four and twelve probe sets were utilized on the grape Affymetrix and NimbleGen microarrays, respectively, with possible cross-hybridization occurring amongst the 18 PN40024 VviERF6Ls [16]. Numerous occurrences of probe cross-hybridization for NimbleGen microarrays of the $V$ viERF6L genes were previously determined [16] (Additional File 20), making it important to consider these results in terms of the $V v i E R F 6 L$ clade response as opposed to individual gene responses. With the shortread length of the RNA-Seq data sets analyzed here and the high homology of the VviERF6Ls, it is unclear how distinguishable $V v i E R F 6 L s$ are individually in the RNASeq analysis.

Data series are referred to as in original publications. SRP117281, PRJNA516950, GSE67191, GSE62744, and GSE62745 were chosen as representative RNA-Seq data series of abiotic stress, berry development, and biotic stress to re-analyze with the V3 structural annotation of PN40024. The data series selected for re-analysis with the V3 structural annotation of PN40024 were used for weighted gene co-expression network analysis (WGCNA) to identify genes that share the same expression pattern as the VviERF6Ls under various stress conditions and developmental stages. All other data series demonstrated in the figures were graphed based on the original author's transcript abundance quantification and statistics.

\section{VviERF6L genes are involved in multiple abiotic stress responses VviERF6L genes respond to water deficit and salinity} $V v i E R F 6 L s$ were differentially expressed in response to numerous abiotic stresses including water deficit and salinity. VviERF6Ls were significantly differentially expressed in CS leaves exposed to rapid dehydration for $1 \mathrm{~h}$ (Fig. 4) [GSE78920] [17]. The VviERF6Ls shared the

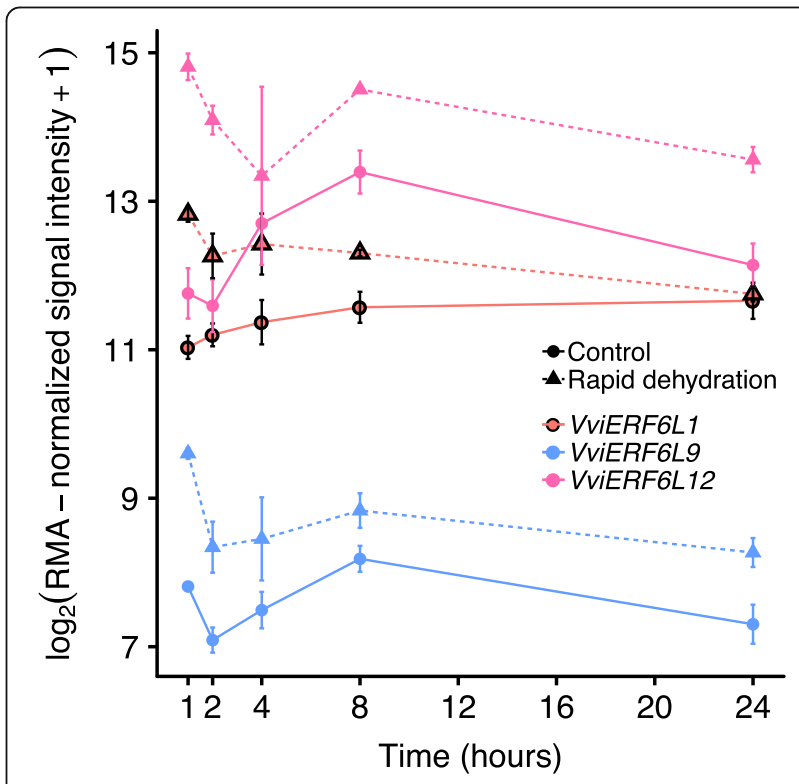

Fig. 4 VVIERF6L gene expression in response to rapid dehydration CS leaves. $\log _{2}(R M A$-normalized signal intensity+ 1 ) gene expression of three VViERF6Ls in CS leaves treated with control (solid lines and circles), or detached and allowed to rapidly dehydrate under regulated conditions (dotted lines and triangles) for 1, 2, 4, 8, or 24 h. VViERF6L1 in red with black outline, VviERF6L9 in blue, and VViERF6L12 in pink [GSE78920]; mean \pm SE 
same general expression pattern in response to rapid dehydration with a significant increase in transcript abundance early in the experiment followed by decreased transcript abundance and plateauing thereafter. VviERF6L12 and 9 demonstrated the highest and lowest levels of expression respectively. VviERF6L1 was among the most responsive $V v i E R F 6 L s$, increasing quickly to the severe stress within $1 \mathrm{~h}$ of treatment and decreasing at all time points after that to eventually be at the same level of expression as control plants after $24 \mathrm{~h}$ of treatment. VviERF6L1 was chosen as a representative gene of the VviERF6L clade for subsequent RT-qPCR and overexpression experiments. RT-qPCR was performed for VviERF6L1 on CS leaves treated with $10 \mu \mathrm{M}$ Protone (sABA). VviERF6L1 transcript abundance in CS leaves did not respond to ABA treatment (Additional File 21), indicating the water deficit response may follow an ABA independent pathway.

CS shoot tips exposed to a severe 16-day salt and water deficit treatment also show significantly increased VviERF6L transcript abundance (Fig. 5) [37]. Probe sets 1618661_s_at (VviERF6L12) and 1619390_at (VviERF6L11) were highly induced by extreme water deficit and salt stress, but 1613698_at (cross-hybridizes to VviERF6L2 and VviERF4) and 1613799_at ( $V v i E R F 6 L 3$ ) were not induced. The accumulation of VviERF6L transcripts on day 16, the point at which both abiotic stresses were most severe indicate some

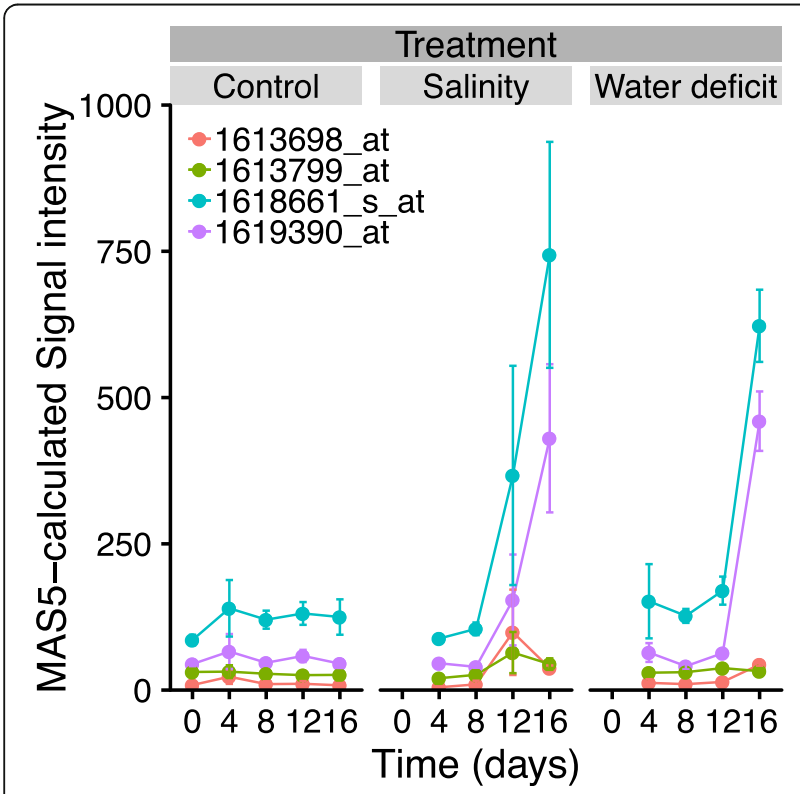

Fig. 5 V ViERF6L gene expression in response to salinity and water deficit in CS shoot tips. MAS5-calculaed signal intensity of four VviERF6L probes in which CS vine were treated with a control nutrient solution, a progressive ramp of $\mathrm{NaCl}+\mathrm{CaCl}_{2}$, or a natural dry down for 16 days [GSE31677]; mean \pm SE
VviERF6Ls may play a role in extreme salt and water deficit responses in grapevine.

VviERF6L differential expression in response to water deficit is supported by recent more comprehensive RNA-Seq data in which four Vitis species (Vitis vinifera cv. CS, Vitis champinii cv. Ramsey (RA), Vitis riparia cv. Riparia Gloire (RI), and Vitis vinifera $x$ Vitis girdiana cv. SC2 (SC)) were treated with well-watered and moderate water deficit (WD) conditions in the form of a natural dry-down for 2 weeks [38]. The grapevines demonstrate significantly differential $V v i E R F 6 L$ expression patterns within each species (Fig. 6). For example, VviERF6L1 is not expressed in SC, but it is expressed in the three other species. Within each Species x Organ x Treatment group, the $18 V v i E R F 6 L s$ follow similar expression patterns to each other (Fig. 6). VviERF6Ls were differentially expressed in leaves and roots in response to the WD (Fig. 6). VviERF6Ls were significantly more highly expressed in roots than in leaves. Consistently, the $V$ viERF6Ls have higher expression in roots than leaves under both Control and WD conditions (13 average TPM (standard error of the mean $(\mathrm{SEM})= \pm 1.5$ ) vs 4.6 average TPM $(S E M= \pm 0.51)$ for roots and leaves, respectively). As a general trend VviERF6L transcripts were decreased in response to WD (e.g. VviERF6L1). The majority of $V v i E R F 6 L s$ have relatively low expression levels apart from VviERF6L12 that demonstrated a significantly higher level of expression (Z-score for two population proportions $p<0.00001)$. VviERF6L8 was consistently the lowest expressed VviERF6L across organs and treatments. Interestingly, leaves from RA (a drought tolerant rootstock originating from Texas, USA) had a significantly higher accumulation of VviERF6L12 transcripts in week 2 WD relative RA Control leaves as well as compared to 2 WD treatment leaves of the other three species. The other species, which are more drought sensitive, did not exhibit an increase or as high of an increase in VviERF6L12 transcripts in the leaves in response to 2 weeks of WD.

Amongst the VviERF6L clade, DEA showed VviERF6L1 and 18 were the most common DEGs in DEA contrasts of interest. DEA was performed for each genotype for WD vs. Control and for each WD treated species to the others for weeks one and two for roots and leaves individually. A list of DEA contrasts of interest for this data set are located in Additional File 22. The frequency at which each VviERF6L was a DEG in the DEA contrasts of interest was quantified (Additional File 23). VviERF6L1 was identified as the most responsive $V v i E R F 6 L$, being a DEG in 14 DEA contrasts of interest followed by VviERF6L18 (a DEG in 10 DEA contrasts of interest); however, both genes were expressed at relatively low levels (Fig. 6). The other VviERF6Ls varied in DEG frequency in the DEA 


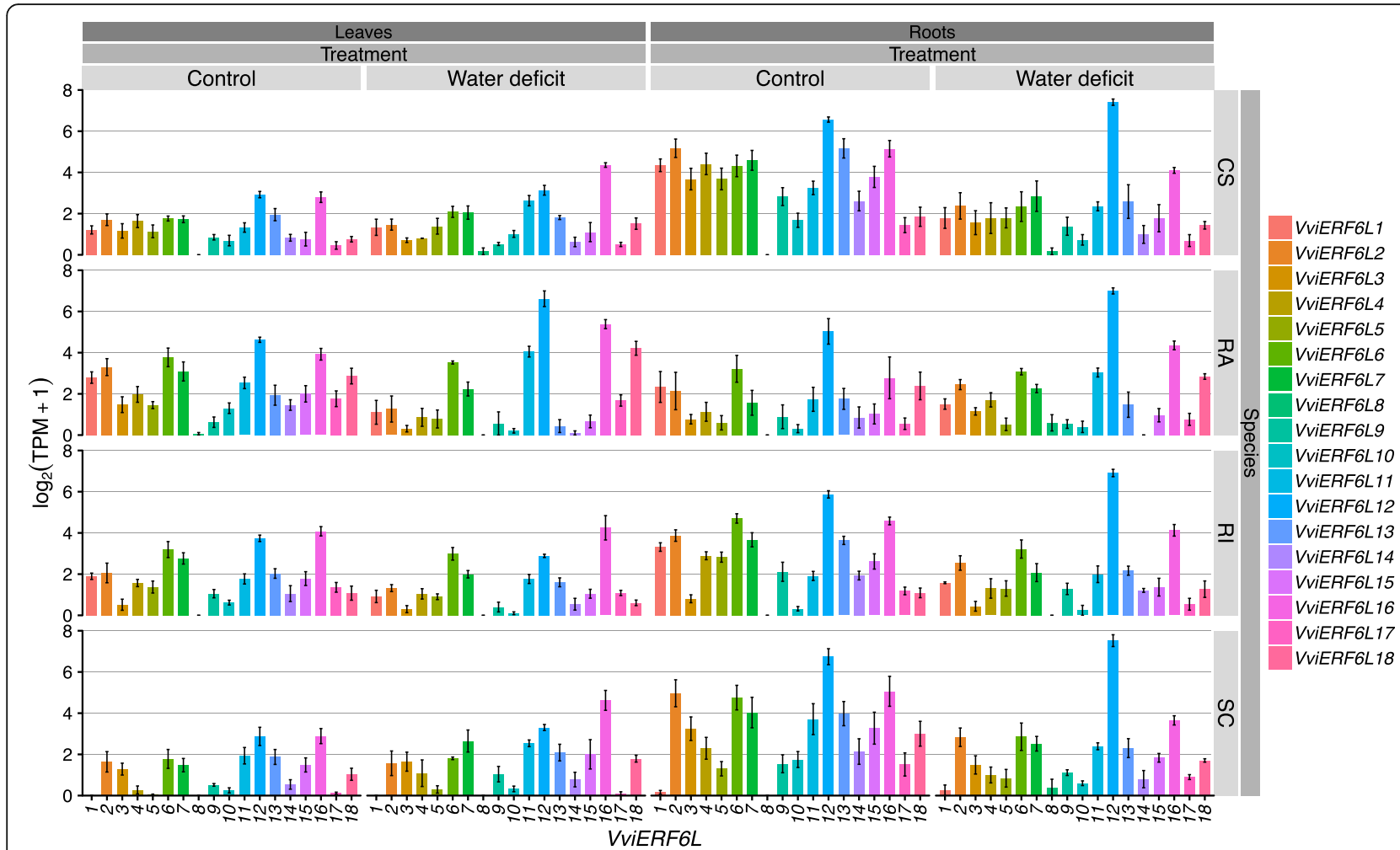

Fig. 6 VViERF6L gene expression in response to 2 weeks of water deficit in leaves and roots of four Vitis species. Log 2 (Transcripts per million $($ TPM $)+1$ ) of 18 V viERF6Ls treated with well-watered control and water deficit for 2 weeks in leaves (left) and roots (right) in CS, RA, RI, and SC [PRJNA516950]; mean \pm SE

contrasts of interest (Additional File 23). VviERF6L7 and 8 were not DEGs in any contrast of interest. The range of frequencies each VviERF6L was a DEG in the DEA contrasts of interest was consistent with the results of the promoter analysis, indicating that while these genes are highly conserved, they are under distinct regulation.

\section{VviERF6L genes respond to chilling and cold}

$V v i E R F 6 L s$ were differentially expressed in leaves in response to chilling, cold, and freezing in the meta-data analysis. Many of the VviERF6Ls responded with analogous expression patterns. Recent RNA-Seq data in which five Vitis vinifera cultivars (Cabernet Franc, Chardonnay, Riesling, Sangiovese, and Tocai Friulano) were treated with chilling (ACC), freezing (FRZ) or a chilling acclimation followed by the freeze treatment $(\mathrm{A}+\mathrm{F})$ demonstrate significant VviERF6L differential expression (Fig. 7) [39]. As with WD response, various $V$. vinifera cultivars demonstrated unique VviERF6L expression in leaves in response to cold treatments. For example, comparing freezing vs. control DEA across cultivars, Chardonnay had the highest significant increase in VviERF6L1 transcript abundance while Tocai Friulano and Sangiovese did not demonstrate ERF6L1 differential expression (Fig. 7 and DEA from original publication [39]). All cultivars had a decrease in transcript abundance of VviERF6L1 in the chilling vs. control treatment comparison (Fig. 7). The transcript abundance of VviERF6L11 and VviERF6L12 was increased in all genotypes in response to the freezing treatment (Fig. 7). The results in Fig. 7 are supported by microarray data in which VviERF6Ls were differentially expressed in CS shoot tips exposed to a chilling treatment for 0,4 , and 8 h (Additional File 24) [40]. To confirm these results, RT-qPCR was performed on VviERF6L1 for RA, RI, CS, and SC whole canopy and single leaves treated with $4{ }^{\circ} \mathrm{C}$ chilling for $0-2 \mathrm{~h}$. In contrast to the previous freezing and chilling treatments, these chilling experiments did not result in a significant difference of VviERF6L1 transcript abundance relative to control; there was however, a significant increase in $C B F 1$ transcript abundance used as a positive control in chilled samples (Additional File 25). It is possible VviERF6L1 was not the most responsive VviERF6L in these species under these conditions. Together these examples from the meta-data analysis reveal $V v i E R F 6 L s$ are differentially expressed with complex responses to temperature reduction in cultivar-, temperature- and time-dependent manners and may play a role in cold response in grapevine. 


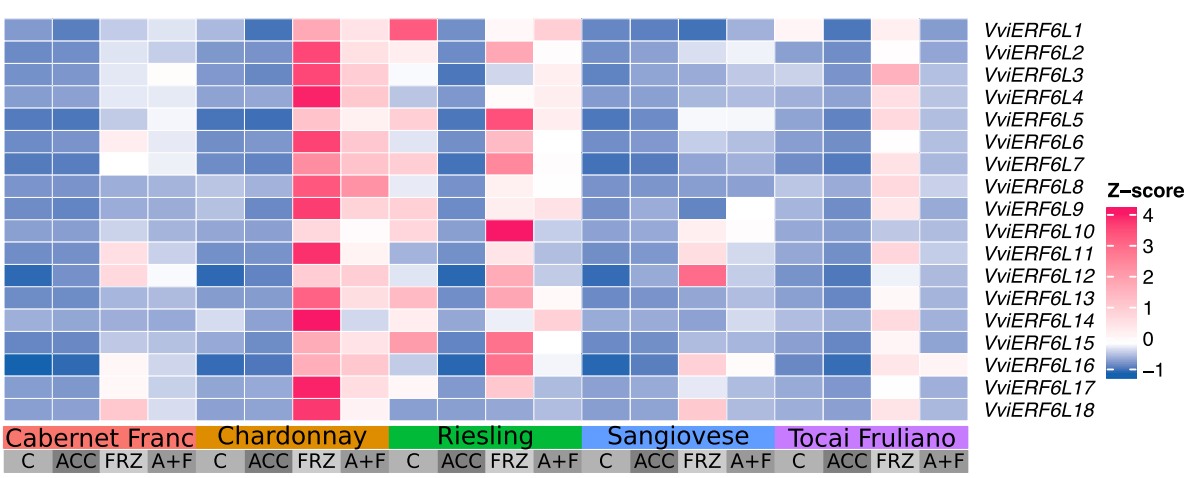

Fig. 7 Heatmap representation of the gene expression of VViERF6Ls in response to cold. Average TPM of 18 VviERF6Ls were $\log _{2}$ transformed and represented as Z-scores (calculated per gene) with pink representing higher and blue representing lower values from leaves of whole vines treated with chilling acclimation (ACC), acclimation followed by freezing (A + F), control (C), and freezing (FRZ) from Cabernet Franc (pink), Chardonnay (orange), Riesling (green), Sangiovese (blue), and Tocai Friulano (purple) [SRP117281]

\section{VviERF6L genes respond to light intensity}

VviERF6Ls were significantly differentially expressed in response to increased light exposure. CS berries exposed to varying light intensity through leaf removal or leaf movement at veraison demonstrated reduced VviERF6L12 transcript abundance in de-seeded berries (pulp and skin only) at late veraison and harvest. The majority of the other VviERF6Ls (all with lower transcript abundance than VviERF6L12) had enhanced transcript accumulation at harvest (particularly with leaf removal) relative to control conditions in which no leaves were (re) moved [41] (Fig. 8) [GSE121146]. In this experiment, leaves were cut off vines for the leaf removal treatment and physically bound in place for the leaf moving treatment. These actions may have elicited a wounding response. However, as the berries remained intact on the vine and only the leaves were removed, it is likely that the increased VviERF6L transcript abundance in the berries is associated with increased light exposure and not a wounding response. The accumulation of VviERF6L transcripts with enhanced light exposure at harvest in combination with the abundance of VviERF6L promoter motifs associated with light response indicates $V v i E R F 6$ Ls may play a role in grapevine response to light intensity. Supportive of these data and VviERF6L light response, VviERF6Ls were also differentially expressed in berries grown under a double cropping system with summer and winter harvests [GSE103226] [42]. In the summer, CS berries grown in this system had the highest level of VviERF6L expression at the end of veraison

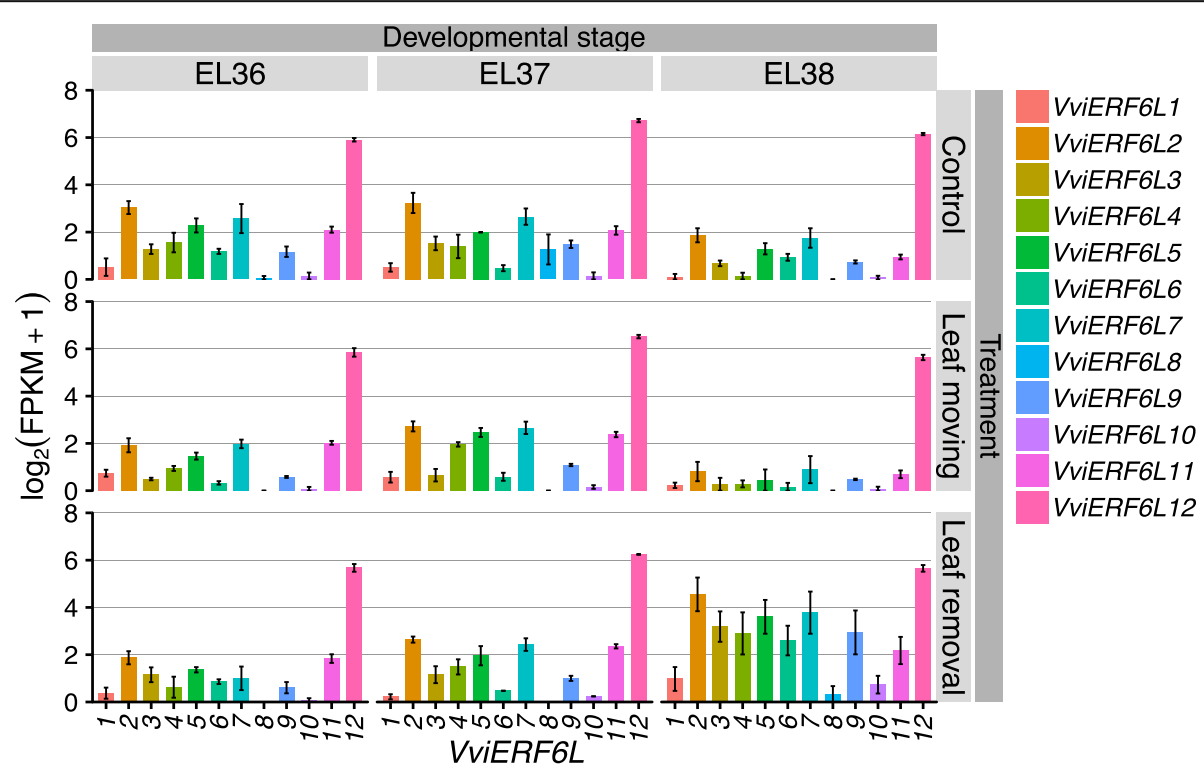

Fig. 8 VviERF6L gene expression in response to light exposure. $\log _{2}(F P K M+1)$ gene expression of 12 VviERF6Ls from berry pericarp at three stages of ripening (EL36-38) under control, leaf movement, and leaf removal light exposure treatments [GSE121146]; mean \pm SE 
(EL36), while there was a distinct depression in VviERF6L transcript level for winter berries (Additional File 26). These data sets support the hypothesis that VviERF6Ls have a cultivar-specific response to abiotic stress and may play a role in response to light intensity.

\section{VviERF6L genes are involved in various biotic stress responses}

VviERF6Ls are differentially expressed in response to Neofusicoccum parvum. CS plants inoculated with $N$. parvum had significantly enhanced VviERF6L transcript accumulation in woody stems 2 weeks after treatment (Fig. 9) [GSE97900] [43]. Interestingly, VviERF6Ls also responded to the wounding aspect of this treatment, which consisted of taking a power drill to the woody stem. The wounding response remained significant for the majority of VviERF6Ls up to 2 weeks after the treatment (Fig. 9).
VviERF6L12 and 8 consistently demonstrated the highest and lowest expression levels, respectively (Fig. 9).

In general, $V v i E R F 6 L$ expression levels significantly increased in response to $E$. necator inoculation in leaves of $V$. vinifera cv. Carignane and six partially resistant Asian accessions (DVIT3351.27 (DVIT3351), Hussiene, Karadzhandal, Khalchii, O34-16, Sochal, and Vavilov) [GSE67191] [44]. The cultivars showed similar expression patterns with differences in expression levels of the VviERF6Ls (Additional File 27). VviERF6L12 and 16 generally had the highest expression in response to the powdery mildew inoculation (Additional File 27). VviERF6L8 generally had low, but detectable expression with the exception of DVIT3351 in which VviERF6L8 had higher expression levels, similar to those of VviERF6L3-7, and Vavilov that did not demonstrate any $V v i E R F 6 L 8$ expression at either time point or treatment. It is possible VviERF6Ls play diverging roles in response to various pathogens.

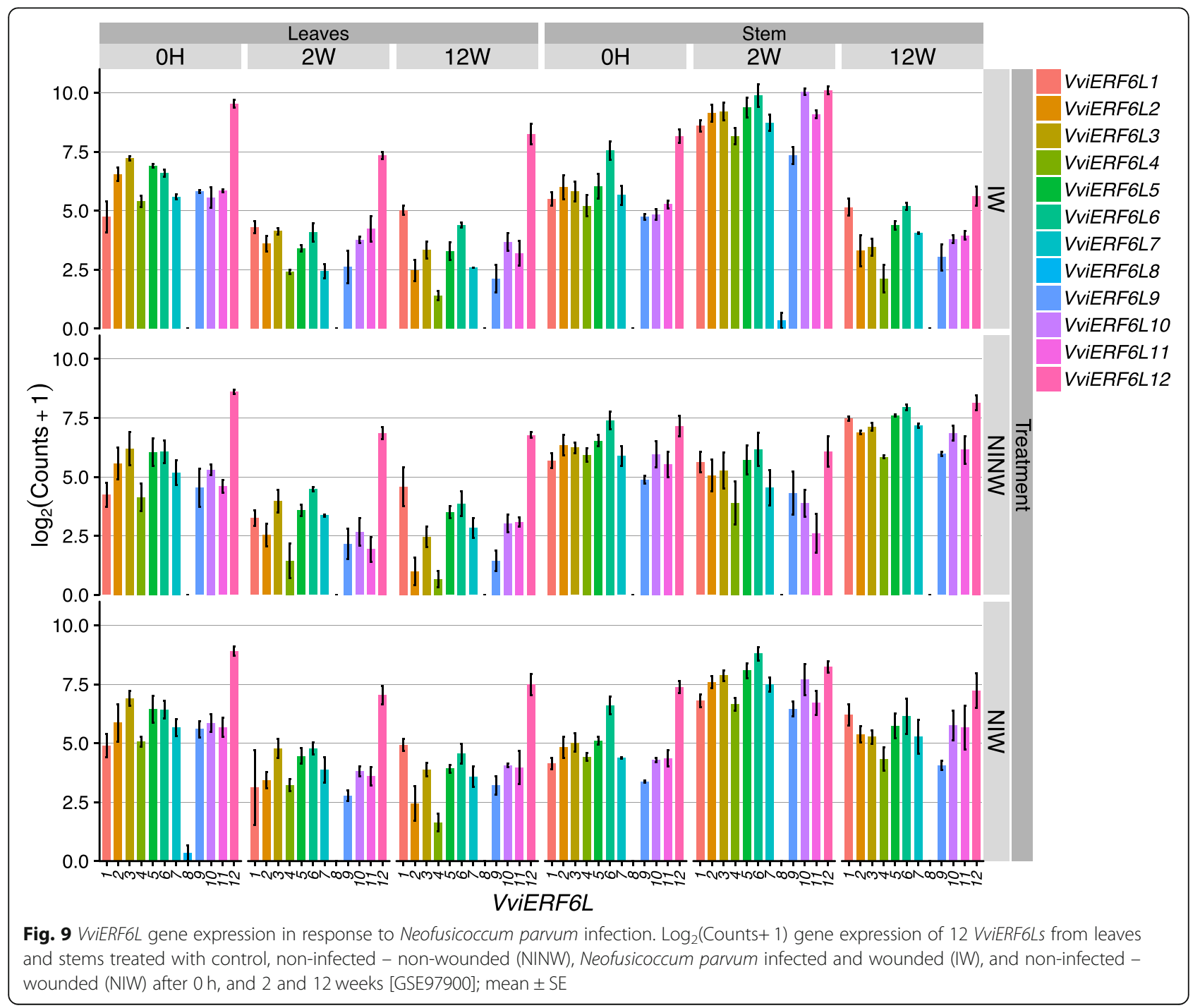




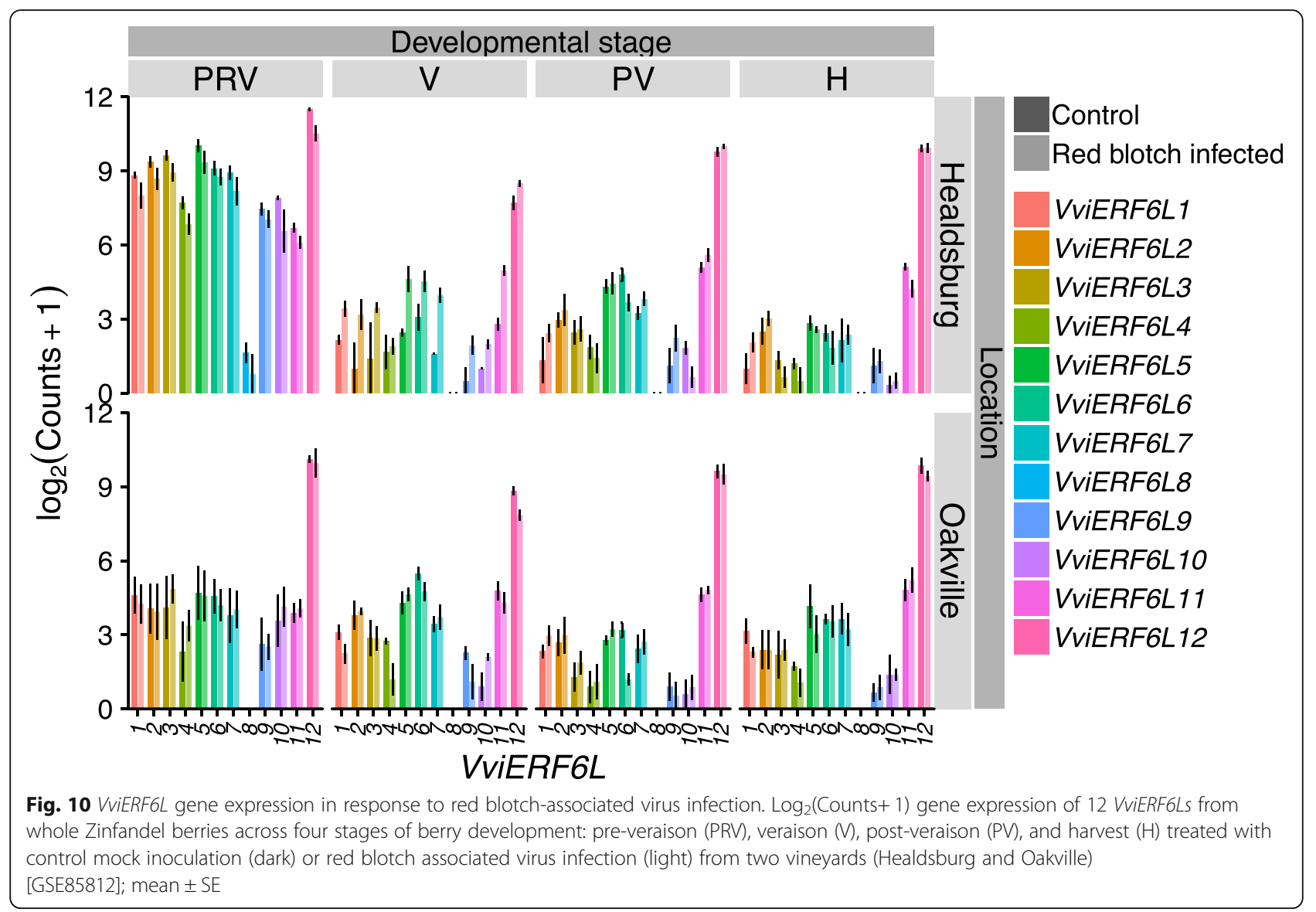

Whole Zinfandel berries had high levels of VviERF6L12 counts across berry development in both control and red blotch-associated virus treatment in two separate vineyards (Fig. 10) [GSE85812] [45]. In control berries, the expression of the VviERF6L clade was highest in the pre-veraison (PRV) stage with subsequent decline in transcript abundance as the berry maturity stage increased. VviERF6L8 expression was only detectable in one vineyard at pre-veraison; in all other cases it does not appear to be expressed (Fig. 10). VviERF6L expression at pre-veraison significantly decreased in response to red blotch-associated virus in at least one vineyard (Fig. 10) [45]. VviERF6Ls showed variable expression across the vineyards in response to red blotch-associate virus particularly at pre-veraison. VviERF6L5, 7, and 10 had increased counts in infected samples at veraison (Fig. 10). At post-veraison, VviERF6L1, 5, and 11 had higher expression in red blotch-associated virus samples in both vineyards, and at harvest, VviERF6L3 had lower counts in infected berry tissue (Fig. 10). While various $V v i E R F 6 L s$ were significantly differentially expressed in response to these pathogens (Figs. 9, 10 and Additional File 27), the pattern and degree of expression across genotypes was not consistent.

\section{VviERF6L genes are involved in berry development}

Unlike abiotic and biotic stress responses, $V v i E R F 6 L$ gene expression patterns and levels show general conservation across cultivars with VviERF6L12 consistently having the highest transcript abundance in red and white berries [46]. One study examining red and white berry development over four developmental stages (pea sized (Pea), touching (Touch), softening (Soft), and harvest (Harv)) showed differential expression of VviERF6Ls across berry ripening, but at a low expression level (Fig. 11). VviERF6Ls had the highest number of VviERF6L transcripts at the pea-sized and touching stages of berry development. VviERF6L transcript abundance decreased as berries softened and was even lower at harvest (Fig. 11). VviERF6L2, 12, and 13 were among the highest expressed VviERF6Ls in white berries with the addition of $V v i E R F 6 L 5,15$, and 16 for red cultivars in the early stages of berry development. In the later stages of berry development, VviERF6L12 clearly had the highest transcript abundance (Fig. 11). From pea-sized to touching berries, VviERF6L8 was expressed in white berries (except Passerina) to a comparable level with other VviERF6Ls, including VviERF6L7 and 9. At all other developmental stages, VviERF6L8 was negligibly 


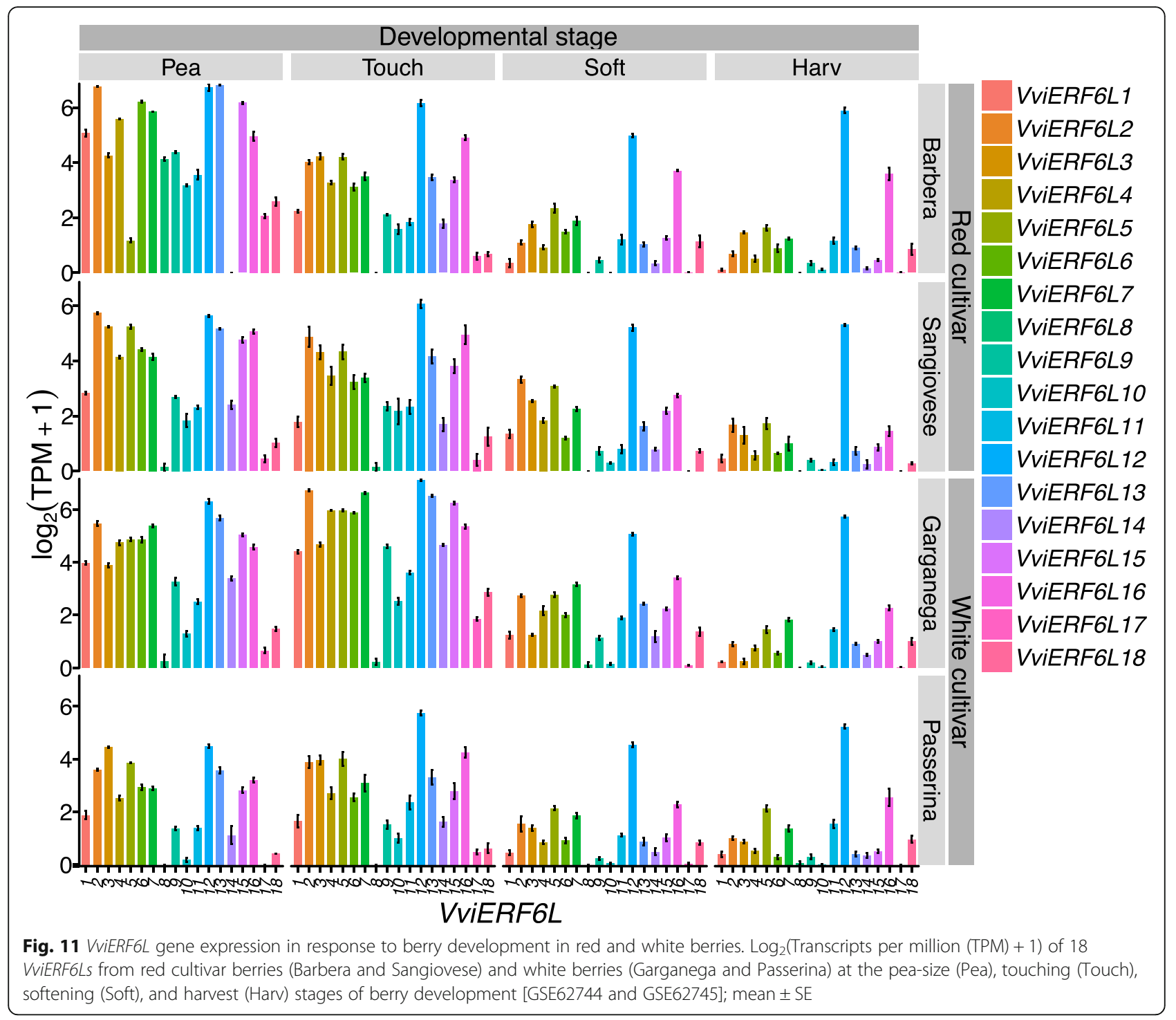

expressed. In red berries, VviERF6L8 was only expressed in Barbera in pea-sized berries. VviERF6L expression across berry development is also consistent across vineyards and years [GSE97578] [47] [GSE41633] [48] (Additional Files 28 and 29). VviERF6L signal intensity peaked significantly at pre-veraison and generally declined as berries approached full ripening (FR) (Additional File 28). There were subtle changes in signal intensity level over the years and vineyards (Additional File 29), but generally expression levels were similar and the VviERF6L expression pattern remained conserved, indicating these genes may not be strongly influenced by environment during berry development.

\section{VviLISCL3 and VviCML45 were the most connected genes} to the VviERF6Ls

Two genes share a similar expression pattern as the 18 VviERF6Ls across various cultivars, organs, and treatments. The meta-data analysis was completed with a gene co-expression analysis to identify genes sharing expression patterns for all VviERF6Ls as a clade between the five data series that were re-analyzed with the V3 annotation of PN40024 (SRP117281, PRJNA516950, GSE67191, GSE62744, and GSE62745). The top 100 genes most connected to each $V v i E R F 6 L$ were extracted from the TOM (Topological Overlap Matrix) for each WGCNA. Common co-expressed genes were identified by comparing these sets of genes. In total, two genes were identified in all five data series that were the most connected to the VviERF6L clade across the various conditions and variables of each data series (Additional File 30). The two co-expressed genes were a Scarecrow-like transcription factor, VviLISCL3 (Vitvi06g00489), and a calmodulin-like protein, VviCML45 (Vitvi14g01975). There were several other genes that shared expression patterns with the VviERF6L clade in 


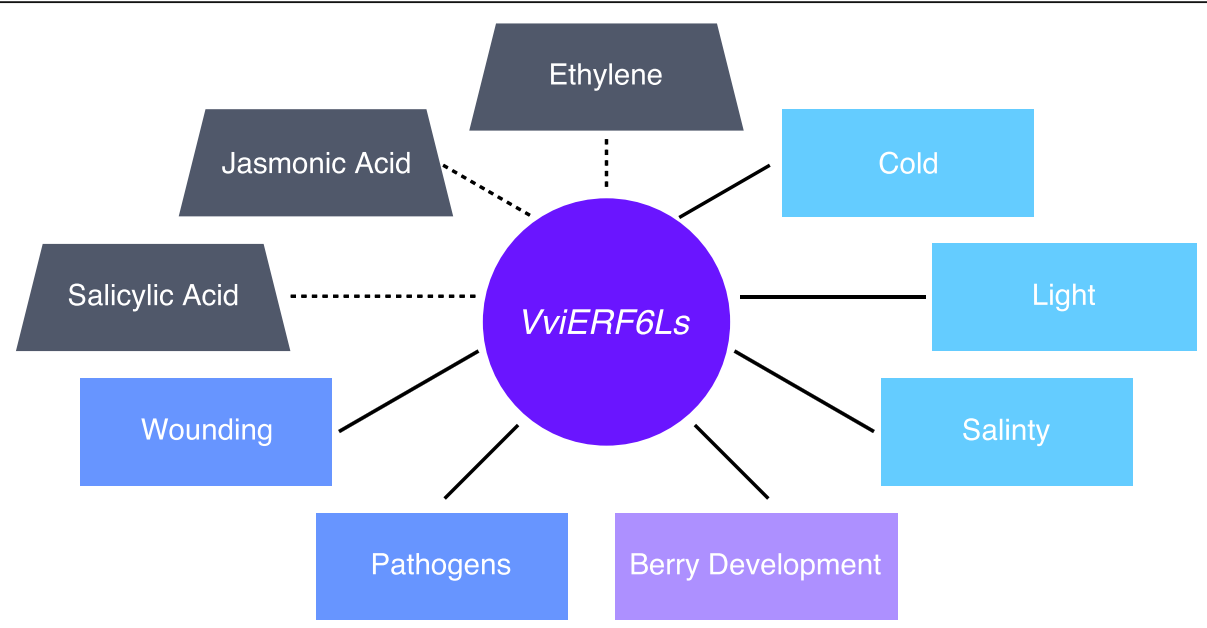

Fig. 12 VviERF6L clade transcriptional response model. A summary model of conditions VviERF6Ls transcriptionally responded to in the meta-data analysis are connected with solid lines. Conditions linked to those investigated in the meta-data analysis requiring further confirmation are connected with dotted lines. Phytohormones are shown in grey. Developmental stages are shown in purple. Abiotic stresses are shown in light blue, and biotic stresses are shown in darker blue

four out of the five data series including VviERF1 and $V v i$ WRKY33 (Additional File 31). The full list of genes co-expressed with the VviERF6L clade in four of the five data series is in Additional File 31. Six of the 16 genes sharing the VviERF6L expression pattern were unannotated.

After analyzing the VviERF6L clade as a whole, the coexpression analysis was repeated for each VviERF6L individually. Surprisingly, no gene was connected to any VviERF6L in all five data series in this individual analysis, not even the other VviERF6Ls. Because no genes were co-expressed with any VviERF6 L individually, genes co-expressed in four out of the five RNA-Seq data series were considered. VviWRKY33 was the only gene to be co-expressed in four out of the five data series in this individual VviERF6L gene co-expression analysis but only for VviERF6L11 and 16. The low number of genes coexpressed with VviERF6Ls in all five RNA-Seq series (two for the clade co-expression analysis (Additional File 31) and zero for the individual VviERF6L coexpression analysis) may be a result of the diverse RNASeq series utilized that examined various organs, genotypes, developmental stages, and stresses.

\section{Summary of the meta-analysis}

Generally, VviERF6Ls were lowly expressed in all data sets, but these genes demonstrate striking fold changes in expression levels and significant differential expression under numerous conditions. VviERF6Ls are broadly expressed across grapevine organs, tissues and in response to various abiotic and biotic stresses as well as throughout berry development (Fig. 12). VviERF6Ls appear to increase in expression in response to severe water deficit and salinity. However, over more long-term moderate water deficit, VviERF6Ls are generally decreased. VviERF6Ls have distinct differential expression in response to cold and light in different cultivars. VviERF6Ls are differentially expressed in response to various pathogens, but the level of expression varies depending on the pathogen and cultivar. VviERF6Ls are also differentially expressed across berry development with the highest expression levels as berries transition into veraison. VviERF6L expression patterns are highly conserved across cultivars, vineyards, and years. The broad range of VviERF6L expression across tissues and expression patterns are conserved throughout numerous data series. The transcriptional response for each member of the VviERF6L clade was dependent on a number of factors (organ, time, treatment, duration, severity, genotype, etc.). The transcriptomic response for each experimental design, while generally conserved, was unique for each VviERF6L (i.e. some members of the clade increased in transcript abundance, some decreased, and others did not respond under a specific condition). The individuality emerging in the VviERF6L clade as well as divergent observations for different severities of similar treatments makes it difficult to generalize common responses. However, a diagram (Fig. 12) was constructed to summarize the conditions that elicited VviERF6Ls responses as well as conditions requiring additional data to further elucidate the role VviERF6Ls play in grapevine. As transcriptomic technology evolves, the VviERF6Ls will be able to be better differentiated and understanding of the clade will be improved.

Two results are clear, first, in all the data series discussed, VviERF6L12, one of the truncated VviERF6Ls, repeatedly demonstrated significantly higher expression than any of the other VviERF6Ls. VviERF6L12 had 2- 
228 times more transcript abundance than the average of all other VviERF6Ls. Across the treatments and conditions of the selected data sets, the transcript abundance of VviERF6L12 averaged 25.6-times more RMAnormalized signal intensity, counts, FPKM, or TPM than the average expression of the other VviERF6Ls (Additional File 32). Second, VviERF6L8 was frequently the lowest expressed $V v i E R F 6 L$ with no detectable expression in certain cultivars. The vast range of VviERF6L expression levels made it necessary to $\log _{2}$ transform the data in the meta-data analysis, so each VviERF6L expression was visible. Although the VviERF6L clade is highly conserved, each $V v i E R F 6 L$ is under unique transcriptional regulation.

\section{Overexpressing VviERF6L1 in Vitis had minor impact on the transcriptome and phenotype}

In previous microarray studies, VviERF6L1 appeared to be the most responsive VviERF6L, with transcript abundance increasing in CS in response to severe leaf dehydration [17] and with changing sugar levels in a study of the late stages of berry ripening [16]. Further investigation of ERF6L1 function was investigated with VviERF6L1 overexpression and knockdown lines. Attempts to establish Vvierf6l1 knock-down lines failed; plants were unable to be re-established after transformation with a T-DNA insertion. An empty vector control (G1) and VviERF6L1 overexpression lines (L12-1, L122, L12-3, L12-11, and L12-23) were created in a Seyval Blanc background under the control of a bi-directional duplex $35 \mathrm{~S}$ promoter fused to EGFP/NPTII in pECBC [49]. Overexpression was confirmed with semiquantitative PCR and RT-qPCR to verify stable overexpression (Additional File 33). Extensive phenotyping revealed VviERF6L1 overexpression lines did not exhibit a morphological phenotype under control conditions, or in response to salinity, water deficit or pathogen spread treatments (Additional File 34). Potential downstream targets of $V v i E R F 6 L 1$ were determined with differential expression analysis on RNA-Seq data from leaves of the empty vector control (G1) and VviERF6L1 overexpression lines (L12-3, L12-11, and L12-23). VviERF6L1 was the only $V v i E R F 6 L$ gene with significantly higher expression in the overexpression lines compared with G1 (Additional File 35). In total, only 14 genes were significantly differentially expressed in all three overexpression lines relative to G1 (Additional Files 36 and 37). Up regulated genes included: VviERF6L1 (Vitvi16g00350), CYP722A1 (Vitvi04g01352), CRK8 (Vitvi11g01160), LAC14 (Vitvi18g01479). Down regulated genes included: three PRB1 (pathogenesis-related protein 1) genes (Vitvi03g00757, Vitvi03g01649, Vitvi03g01651), unannotated genes (Vitvi03g01650, Vitvi07g01985, Vitvi11g01692,
Vitvi18g02319), MET1 (Vitvi12g02119), WAKL1 (Vitvi18g00024), and LIMYB (Vitvi01g01444).

\section{Discussion}

The VviERF6L clade was expanded and conserved

VviERF6L genes with no previously known functions were identified to be an expanded clade in Vitis in comparison with other plant species. Using protein and promoter motifs and a meta-data analysis, this work shows VviERF6Ls are highly conserved proteins. Manual and in silico techniques identified and confirmed 18, 26, 15, and 14 members of the VviERF6L clade in PN40024, CS, $\mathrm{CH}$, and $\mathrm{CA}$ respectively (Additional File 12). The high sequence and spatial conservation of amino acid motifs validates these sequences as members of the $V v i E R F 6 L$ clade in the AP2/ERF subfamily IX. The small differences in protein motif sequence and position may contribute to the differential regulation of the VviERF6Ls observed in the publicly available microarray and RNASeq analysis. Four known protein domains identified (Fig. 1) in the VviERF6L proteins coincide with those in Arabidopsis ERF IXb transcription factors including ERF5 (At5g47230), ERF6 (At4g17490), ERF104 (At5g61600) and ERF105 (At5g511290) [15]. The VviERF6Ls contain CMIX-2, 5, and 6 domains (putative post-translational modification sites) as well as the AP2/ ERF domain (DNA-binding) (Fig. 1). While VviERF6L1 has highest orthology to AtERF6, it contains an additional domain, CMIX6, found in AtERF104, but not present in AtERF6. This domain is thought to contain a MAP kinase phosphorylation site [15].

Four motifs located near the amino and carboxyl ends of the VviERF6L proteins were unable to be identified. ERF transcription factors interact with numerous other proteins including regulatory enzymes, coactivators, repressors, and other transcription factors [50-52]. These interactions regulate stability and activity as well as localization of ERFs. The unidentified VviERF6L protein domains may play roles in post-translational regulation and/or interactions with other proteins. One such interaction occurs with AtERF104, which is phosphorylated by MPK 6 and released from this interaction in the presence of the flg22-peptide to influence ethylene signaling and pathogen susceptibility [53].

Vitis vinifera had the highest number of VviERF6L paralogs compared to species with the closest related genes (Additional File 5), marking this as an expanded clade in grapevine. The VviERF $6 L$ clade consists of nearly consecutive genes on chromosome 16 . The VviERF6L paralogs likely originate from gene duplications. Duplication events are common and frequent in plants contributing to gene evolution and diversification [54]. Recent whole genome duplications in cotton [55], 
wheat [56], and soybean [57] and dispersed duplication in corn [58] gave rise to agronomically valuable traits of 4 of the top 10 produce crops in the United States. Exploring VviERF6L evolution in wild grapevine as well as the ancestor of domesticated grapevine may provide more evidence and a time line for the hypothesized duplication event. The contiguous VviERF6Ls may be tandem array genes, arising from tandem duplication of an ancestral gene. The significance of tandem ERF duplication was recently described in Fragaria vesca [59]. Tandem FveERFs are differentially expressed from one another in response to abiotic stress, suggesting gene divergence occurring after tandem duplication(s) [59]. A similar event may have occurred with grapevine VviERF6Ls. The possibility of ERF6L duplication in Vitis is supported by the expansive ERF family in pear (155 members) [60], another woody perennial, as well as Arabidopsis (122 members) and rice (139 members) [15].

\section{Individual VviERF6Ls had unique gene expression}

The VviERF6L clade is under unique transcriptional regulation in response to numerous conditions (Fig. 12). Contrasting the high similarity in protein sequence, VviERF6L putative promoter regions (Fig. 3) showed greater diversity than VviERF6L protein sequences (Fig. 1), indicating these genes are under distinct transcriptional regulation. However, strong patterns and conserved motifs were detected across the promoter regions. The CAATBOX1 motif was the most abundant motif in the 18 VviERF6L upstream regions (Additional File 15), which may play a role in tissue specific gene expression [61] and contribute to VviERF6L expression across the broad range of tissues observed in the meta-data analysis. The MYB1AT motif is present in dehydration responsive genes like RD22 [62]. This motif was present in all VviERF6L promoters, but with fewer repetitions, supporting the transcriptomic data that VviERF6Ls are only responding to severe water stress (Fig. 5). DOFCOREZM, another abundantly present cisregulatory element in the $V v i E R F 6 L$ promoter regions (Additional File 15), is the binding motif for Dof proteins, a diverse group of transcription factors with roles in defense and phytohormone responses, light, and development [63]. Interestingly, numerous VviERF6L promoter motifs associated with light responses were identified including the CACTFTPPCA1, DOFCOREZM, GATABOX, GT1CONSENSUS, with responses supported by the results from the meta-data analysis (Fig. 8 and Additional File 26). AtERF5, has also been linked to light responses [64]. It is possible the light response of VviERF6Ls is tissue specific and was not well identified in the berry-centric microarrays and RNA-Seq data sets, requiring further investigation in other tissues. Several motifs associated to biotic stress responses were also present in the promoter including WBOXATNPR1, which had the highest number of repeats in VviERF6L12. Together, the presence of these motifs supports the proposed roles of VviERF6Ls in extreme water deficit, cold, light, and pathogen responses.

The diverse promoter sequences partially explain the distinct VviERF6L expression levels and patterns observed across the RNA-Seq and microarray series. VviERF6L12 had one of the most variable promoter sequences (Additional File 16) along with VviERF6L1 and VviERF6L6. Putative promoter regions of VviERF6L12 (and other VviERF6Ls) contained unique cis-regulatory elements as well as distinctive motif placement and replication. The distinct conditions in which certain VviERF6Ls are DEGs (Additional File 23) in the metadata analysis may be partially explained by the diversity in upstream sequences.

Additional differences in transcriptional regulation may be contributed to by epigenetics. ERFs have been demonstrated to regulate one another and effect epigenetic regulation through the ethylene-responsive element binding factor-associated amphiphilic repression (EAR). The EAR motif is a short peptide sequence comprised of charged and polar residues (LxLxLx or DNLxxP) that are proposed to confer gene silencing via histone modification and chromatin remodeling through an unresolved mechanism $[2,65]$. AtERF3, 4, and 7 contain EAR motifs that act as active repressors of target and reporter genes [66-69]. Epigenetic regulation and upstream effectors of VviERF6L expression require further investigation to determine interactions with EAR containing ERFs.

\section{VviERF6L1 expression was independent of $A B A$ treatment}

The transcript abundance of VviERF6Ls was shown to increase in response to severe osmotic stress and at preveraison in berry develop in preliminary microarrays that originally brought attention to the VviERF6L clade [16, 17]. Although ERFs are traditionally associated with ethylene signaling, these ERF transcription factors are well documented to act as hubs for hormone-crosstalk and signaling integration [9-14]. ABA is a key phytohormone in abiotic stress responses and berry ripening in grapevine. Water deficit responses are ABA-dependent and/or ABA-independent [70]. However, VviERF6L1 expression was not significantly different in CS leaves treated with exogenous ABA relative to control (Additional File 21). Interestingly, AtERF5 is also not associated with ABA signaling [71]. It is possible other VviERF6L transcripts may increase or decrease in response to ABA treatment, as the VviERF6Ls are distinctly regulated, but no known ABRE cis-regulatory elements were identified in the VviERF6L upstream regions (Additional File 15). Alternatively, a $V v i E R F 6 L$ ABA response may be tissue specific (e.g. berries 
transitioning into veraison). The increased expression of VviERF6L1 in response to various abiotic stresses and berry development in the meta-data analysis, but the lack of induction in response to ABA foliar spray indicates the VviERF6L1 abiotic-stressed-based induction may be independent of the ABA signaling pathway. RTqPCR along with microarrays and short-read RNA-Seq were determined to be less than ideal techniques for quantifying VviERF6L transcripts due to the high sequence similarity of these genes; primers and probes could hybridize to (and short reads could be attributed to) multiple $V v i E R F 6 L s$ resulting in inflated transcript levels and difficulty separating the VviERF6Ls independently. With the advent of Iso-Seq and the ability to quantify full-length transcript reads, the future of distinguishing VviERF6Ls individually will be more reliable and accurate [72].

\section{VviERF6Ls were differentially expressed in response to water deficit, salt, and cold}

VviERF6L expression significantly increased in response to extreme water deficit and salt (Figs. 4 and 5). However, exposing CS, RA, RI, and SC vines to a moderate one- and two- week natural dry down revealed ERF6Ls were generally significantly decreased in transcript abundance (Fig. 6). Each VviERF6L had a unique expression level that responded differently in each species and organ examined in this experiment, further distinguishing the VviERF6Ls individually and supporting the hypothesis each $V v i E R F 6 L$ is under specific transcriptional regulation.

The uniqueness of VviERF6Ls across tissues and cultivars is further demonstrated with significant differential VviERF6L expression in response to cold (Fig. 7 and Additional File 24 and 25). Again, each VviERF6L in the different cultivars had varied responses to the cold (Fig. 7). There were also differences between the leaves and shoot tips investigated in the different data series. $V$ viERF6Ls generally followed similar expression patterns within treatments but to different degrees of expression across cultivars and Vitis species indicating differential ERF6L regulation across these division of Vitis. Differences in ERF6L regulation may contribute to differences in abiotic stress tolerance across various grapevine cultivars.

\section{VviERF6Ls were differentially expressed in response to biotic stresses}

Differential expression analysis performed on VviERF6L1 Vitis overexpression relative to control vines revealed three $P R B 1$ paralogs, one putative $P R 1$, and a putative mildew resistance locus that were significantly down regulated in the overexpression lines. PR1 is a common SA signaling marker gene up-regulated in response to certain pathogens including Pseudomonas syringae [73-76]. The distinct downregulation of this gene and its paralogs in VviERF6L1 overexpression lines is consistent with the enhanced susceptibility to Pseudomonas syringae documented in the AtERF6 and AtERF5 overexpressors [77]. It is possible VviERF6L1 works in combination with other TFs to impact grapevine susceptibility to various pathogens. Another DEG in OX VviERF6L1 lines, LAC14, may also be linked to biotic stress response. This gene encodes a laccase that is part of secondary metabolism responsible for lignin degradation or polymerization [78] and was significantly upregulated in the OX VviERF6L1 lines. Lignin biosynthesis and accumulation aids in plant resistance to insect pests. Lignin deposition is also associated with abiotic stress response and antagonization with plant growth $[79,80]$. The duality of the DEGs' roles in both abiotic and biotic stress response further strengthen the hypothesis of broad functionality of VviERF6Ls. $V v i E R F 6 L s$ were differentially expressed in response to various pathogens in the meta-data analysis (Figs. 9, 10 and Additional File 27). The level of VviERF6L expression and specific VviERF6Ls that were DEGs was pathogen and tissue specific.

VviERF6Ls response to pathogens is conserved in Arabidopsis. AtERF5, one of the closest orthologs to the VviERF6L clade, directly interacts with AtERF6 and 8 as well as SCL13 and MPK3 and 6 in unique combinations to respond differentially to Pseudomonas syringae [81], Botrytis cinera [77], Alternaria brassicicola [81], and Meloidogyne incognita [82]. Aterf5/6 double mutants have enhanced susceptibility to $V$. longisporum, a fungus that induces severe wilting and plant death [83]. Another pathogen related example of a potential role in pathogen response shows decreased VviERF6L expression in response to Plasmopera viticola (downy mildew) in more susceptible vines and either an increase or no significant change in transcript abundance in more tolerant vines $[84,85]$.

\section{VviERF6Ls were differentially expressed over the course of berry development}

The VviERF6Ls had a consistent significant differential expression pattern over the course of berry development. VviERF6L transcript abundance was significantly increased at pre-veraison as the berries transitioned into ripening and decreased as the berries approached full ripening and harvest (Fig. 11). The VviERF6L expression pattern over berry development was conserved across red and white berries (Fig. 11), vineyards, and years (Additional Files 28 and 29).

The VviERF6L ortholog Solyc08g078190 identified from the Pan-taxonomic Compara Gene Tree in Gramene follows a similar expression pattern as VviERF6Ls. This gene, annotated as SIERF.B13 [86] or 
ERF1a [87], increases in the breaker stage (equivalent to veraison in grapevine) of berry development in tomato and decreases in transcript abundance as berries ripen [86]. Another VviERF6L tomato ortholog, Sl-ERF.B3 (Solyc05g052030), also plays a role in berry development [88]. Like grapevine, tomato ERFs can have increased or decreased transcript abundance under certain conditions [86]. This similarity between non-climacteric grapevine and climacteric tomato along with the significant transcriptomic responses in the meta-data analysis support a potential role of $V v i E R F 6 L s$ in berry development requiring further investigation.

\section{VviLISCL3 and VviCML45 were genes connected to the VviERF6L clade}

Little is known about VviLISCL3 and VviCML45 that were co-expressed with the VviERF6L clade. VviLISCL3 is a GRAS transcription factor with roles in plant development, abiotic stress, and disease response [89, 90] similar to the VviERF6L expression profile identified from the meta-data analysis. VviLISCL3, like the $V$ viERF6Ls, appears to be ubiquitously expressed across plant tissues with the exception of pollen [91]. VviLISCL3 was differentially expressed over berry development and likely plays a role in berry set and the early stages of berry development [91]. However, unlike the VviERF6Ls, VviLISCL3 had high expression levels at ripe, harvest, and post-harvest stages of berry development [91]. SIGRAS13, the VviLISCL3 ortholog in tomato, shows the same expression pattern and role in fruit ripening [92]. AtCML45, the Arabidopsis ortholog of VviCML45, is differentially expressed in nrp1 nrp2 Arabidopsis double mutants that lack these histone chaperones associated with root growth [93] and may provide a very loose link of VviERF6Ls to epigenetic modification that ERFs are known to play a role in $[2,65]$. The link between VviERF6Ls, VviLISCL3, and VviCML45 was discovered but remains unresolved, requiring further clarification.

\section{Overexpressing VviERF6L1 had a minimal impact on grapevine}

Overexpressing VviERF6L1 in Vitis vinifera did not result in a detected morphological phenotype. This observation may be attributed to the limited number of genes VviERF6L1 overexpression affected (Additional File 30). It is possible a $V v i E R F 6 L 1$ overexpression phenotype is only detectable under specific conditions not tested in this work. VviERF6L1 was the only member of the $V v i E R F 6 L$ clade with enhanced gene expression in the VviERF6L1 overexpression lines (Additional File 35). The other VviERF6L genes may share similar functions and could have been down regulated in response to the overexpression of VviERF6L1. The hypothesis that
VviERF6L genes share similar functions is supported by the redundant gene and promoter sequences of VviERF6L genes (Figs. 2 and 3). Paralog downregulation in response to overexpression is observed in plants. For example, CYP78A8, one of the closest paralogs to CYP78A9, is downregulated in response to CYP78A9 overexpression [94]. However, this does not appear to be the case in the overexpression of VviERF6L1. The promoter and meta-data analysis support this conclusion. Although the VviERF6Ls share similar expression patterns, expression levels and transcriptional regulation are unique for each $V v i E R F 6 L$, and the overexpression of $V$ ViERF6L1 does not appear to influence the transcription of the other VviERF6Ls. Further studies are needed to identify and confirm specific VviERF6L downstream targets.

\section{VviERF6L in grapevine is distinct from Arabidopsis ERFs} Arabidopsis thaliana ERFs do not have strong orthology to VviERF6Ls. AtERF5 and AtERF6 are the closest orthologs to VviERF6L1. AtERF6 and AtERF5 are rapidly induced in growing tissues and effectively arrest cell cycle progression and plant growth in response to osmotic stress [95]. AtERF5 is involved in karrikin signaling [71], water deficit and osmotic stress [96], programmed cell death [97], and immunity response [77]. AtERF6 overexpression lines are hypersensitive to osmotic stress [96]. VviERF6L1 overexpression vines exposed to chilling, water deficit, and salinity demonstrated no significant differences from controls in the reduction in growth, carbon assimilation, or canopy surface area relative to empty vector control plants (Additional File 34). However, VviERF6Ls were shown to respond transcriptionally to osmotic stress (Fig. 5), but no link was made to cell cycle regulation. AtERF6 is also a positive regulator of antioxidant production with Aterf6 mutants having stunted growth and enhanced levels of ROS and anthocyanin content [98]. AtERF6 is an inductor of stress tolerance genes and a key activator of leaf growth inhibition [99]. VviERF6L1 overexpression lines had no reduction in growth or development relative to empty vector controls (Additional File 34), and no connection was made specifically to antioxidants and anthocyanins.

In Arabidopsis, AtERF6 acts as a regulatory hub favoring stress defense mechanisms at the cost of plant growth through DELLA protein stabilization via ethylene and gibberellin crosstalk [99, 100]. VviERF6Ls were found to respond to various pathogens (Figs. 10, 11 and Additional File 27), but no negative impact on growth was found at least in the case of VviERF6L1 (Additional File 34). It is possible other VviERF6Ls could impact growth. VviERF6L1 overexpression lines did, 
however, have significantly decrease transcript abundance of several $P R 1 B$ genes, associated with pathogen stress response (Additional File 36). AtERF6 and AtERF5 function redundantly in response to biotic stresses and act as a point of crosstalk between ethylene and JA signaling, providing enhanced resistance to Botrytis cinera, but increased susceptibility to Pseudomonas syringae in AtERF5 and AtERF6 constitutive plants. Aterf5/ Aterf6 double mutants demonstrate enhanced susceptibility to Botrytis cinera [77]. Preliminary pathogen spread assays did not show significant differences in OX VviERF6L1 leaves relative to empty vector control (Additional File 34). Further investigation of VviERF6L1 overexpression susceptibility to various pathogens is on-going and may reveal a more definite role in biotic stress response.

AtERF6 overexpression lines demonstrate extreme dwarfism [96]. AtERF6 activates AtERF11 transcription, which in turn competes with AtERF6 for DNA-binding sites as a balancing mechanism between stress response and growth [99]. AtERF11 overexpression is able to rescue the dwarf phenotype in AtERF6 overexpression plants [99]. A VviERF6L1-ERF11 antagonism was not detected in the DEA of the VviERF6L1 overexpression lines. It is possible a different VviERF6L is responsible for this regulatory mechanism or that this interaction is not present in Vitis.

AtERF6 is also documented to activate MYB51, WRKY33, and STZ [96], all genes with roles in biotic $[101,102]$ and abiotic [103-105] stress responses. VviERF6Ls are co-expressed with VviWRKY33 (Additional File 31), linking the two species in this signaling pathway, but at least VviERF6L1 does not appear responsible for VviWRKY33 activation (Additional File 34). The distinction of the Vitis ERF6L clade from the closest Arabidopsis orthologs is supported by the work presented here including the lack of comparable phenotypes in overexpression lines and transcriptomic responses from the meta-data analysis. However, while Vitis ERF6L genes are unique, they may functionally overlap with the distant Arabidopsis orthologs to an extent with associated abiotic and biotic stress responses. ERF TFs in Arabidopsis and Vitis are differentially regulated by abiotic stresses [106] including cold, salinity [107], and water deficit as well as biotic stresses such as wounding and pathogen attack $[108,109]$.

\section{Conclusions}

VviERF6Ls are an expanded and highly conserved Vitis clade. VviERF6L expression was increased in berries at the pre-veraison stage and was found to be induced in leaves by extreme abiotic stress including salt, cold, and water deficit (Fig. 12). VviERF6L1 was not induced by
$\mathrm{ABA}$, indicating a role in water deficit responses through an ABA-independent pathway. Overexpression of VviERF6L1 in a Seyval Blanc background did not yield a detectable morphological phenotype, emphasizing the separation of this clade from the Arabidopsis orthologs ERF6 and ERF5, overexpression of which results in extreme dwarfism and osmotic stress sensitivity. DEA performed on RNA-Seq from the VviERF6L1 overexpression lines identified 14. DEGs involved in abiotic and biotic stress responses. Overall, VviERF6Ls have versatile functions and are expressed in numerous tissues in response to abiotic and biotic stress and may play multiple roles in these processes that require further elucidation.

\section{Methods}

Phylogenetic analysis and cis-regulatory element identification of the ERF6-like clade

DNA sequences from the VviERF6-like clade and Arabidopsis thaliana orthologs were obtained from ORCAE and Araport11 and compared to CRIBI and TAIR identifiers respectively $[18,110-112]$. Sequences were aligned with MUSCLE using the msa $\mathrm{R}$ package and a phylogenetic tree was drawn using a clustal omega alignment in Mega X [31, 113]. A Maximum likelihood method and Jones-Taylor-Thornton matrix-based model with Bootstrapping $n=1000$ replicates were used to create a consensus tree consisting of PN40024, CS, CH, and CA VviERF6Ls and PN40024 ERFs. Branches present in < $50 \%$ bootstrap replicates are collapsed. The initial tree for the heuristic search was generated from the maximum parsimony method. The Subtree-PruningRegraftings - Fast (SPR level 3) was used. Conserved motifs were identified and confirmed in MEME using the standard settings (motif limit at 50 amino acid residues) [22, 114]. Motifs were characterized with InterPro and modeled in SWISS-Model using standard settings $[24,25]$. VviERF6L1 gene upstream regions $(-3000 \mathrm{bp})$ were obtained with the $\mathrm{R}$ package GenomicFeatures [115]. Cis-element enrichment and identification analysis was performed with PLACE [32]. Promoter regions were aligned, and a phylogenetic tree was made in clustal omega.

\section{Meta-data analysis}

RNA-Seq and microarray data sets were downloaded from NCBI GEO [35] and SRA [36] with GEOquery [116] version 2.50.5 and the SRA Toolkit version 2.9.2, respectively. Data series that were re-analyzed with the V3 PN40024 annotation were quality checked with fastqc [117] and trimmed with trimmomatic version 0.35 [118]. Transcript abundance was quantified with Salmon version 0.10.1 [119] using quasi-mapping, seqBias, gcBias, fldMean 50, fldSD 1, validateMappings, libType 
A, and rageFactorizationBins 4. Tximport version 1.10.1 was used to generate the count matrix. Differential expression analysis was performed with DESeq2 version 1.22.2 [120]. Co-expression analysis was performed using WGCNA version 1.68 for all $18 V v i E R F 6 L s$ as a clade as well as for each $V v i E R F 6 L$ individually using the five data series that were re-analyzed with the V3 annotation of PN40024. The top 100 genes most connected to the VviERF6L clade was used to make a Venn diagram (Additional file 30).

\section{Plant transformation}

VviERF6L1 CDS was inserted into pECBC under the control of a bi-directional duplex $35 \mathrm{~S}$ promoter fused to PR1 and EGFP/NPTII [49]. Seyval Blanc cell cultures were transformed with Agrobacterium tumefaciens and grown under kanamycin selection. The empty vector was also inserted into cells to be used for control plant generation. Transgenic lines were created at the MidFlorida Research and Education Center for the Institute of Food and Agricultural Sciences.

Transgenic cells were confirmed with GFP screening performed with confocal microscopy. Four overexpression lines (L12-1, L12-2, L-12-3, L12-11, and L12-23) and one control line (G1) were regenerated into full plants grown under greenhouse conditions.

\section{Plant materials and growth conditions}

Own-rooted Vitis vinifera (L.) cv. Cabernet Sauvignon clone 8 (CS) were obtained from Inland Desert Nursery (Benton City, Washington, USA). Vitis champinii cv. Ramsey (RA), Vitis riparia cv. Riparia (RI), and Vitis vinifera $x$ girdiana SC2 (SC) were obtained from the Plant Foundation Services at UC Davis (Davis, CA USA). Mature plants of the five transgenic lines and of the four genotypes CS, RA, RI and SC were grown in Stuewe and Son's tree pots TP915R $(22.9 \mathrm{~cm} \times 39.4 \mathrm{~cm})$ containing 1 : 1:1:2 perlite:peat moss: Grow Mulch (Kellogg):washed sand. Each pot contained $\sim 8.0 \mathrm{~kg}$ of soil mix. Mature plants were irrigated with $\sim 1.2 \mathrm{~L}$ of $\mathrm{pH} 5.5$ water biweekly. Propagates were generated from single node cuttings of mature plants and transferred in trays containing pH 5.5 water with an air-stone until roots emerged. Plants were transferred to Stuewe and Son's Anderson AB39 pots $(7.3 \mathrm{~cm} \times 22.9 \mathrm{~cm})$ consisting of $1.0 \mathrm{~kg}$ quikrete medium grain sand and $\sim 40 \mathrm{~g}$ of 50:50 perlite-vermiculite mix. Plants were covered for 1 week to increase relative humidity and slowly acclimated to greenhouse humidity conditions over the course of 2 weeks. Greenhouse conditions were maintained at approximately $21-26.5{ }^{\circ} \mathrm{C}$ and $20-50 \%$ relative humidity. All pots were elevated $7.5 \mathrm{~cm}$ off the floor with perforated black plastic flats. Light was supplemented with $1000 \mathrm{~W}$ high pressure sodium light bulbs approximately
$4.5 \mathrm{~m}$ above the floor directly over the center of the experimental area. Supplemental light had a 16:8-h lightdark cycle. Light intensity of the greenhouse averaged $1200 \mu \mathrm{E} \mathrm{m} \mathrm{m}^{-2} \mathrm{~s}^{-1}$. Propagates were irrigated every other day (until they reached approximately $70 \mathrm{~cm}$ height at which point the experimental treatment began) with Cramer's complete nutrient solution $(1.5 \mathrm{mM} \mathrm{Ca}$ $\left(\mathrm{NO}_{3}\right)_{2}, 2 \mathrm{mM} \mathrm{KNO}{ }_{3}, 0.6 \mathrm{mM} \mathrm{MgSO}, 1 \mathrm{mM} \mathrm{KH} \mathrm{PO}_{4}$, $1.5 \mathrm{mM} \mathrm{CaCl}_{2}, 36 \mu \mathrm{M} \mathrm{\textrm {Fe } _ { 2 }}{ }^{+}$Sprint 330, $1 \mu \mathrm{M} \mathrm{MnSO}$, $0.5 \mu \mathrm{M} \quad \mathrm{CuSO}_{4}, 20 \mu \mathrm{M} \quad \mathrm{ZnSO}_{4}, 20 \mu \mathrm{M} \quad \mathrm{H}_{3} \mathrm{BO}_{3}$, and $\left.0.01 \mu \mathrm{M}\left(\mathrm{NH}_{4}\right)_{6} \mathrm{Mo}_{7} \mathrm{O}\right)$.

\section{Phenotypic characterization of VviERF6L1 overexpressing lines}

Mature transgenic plants were grown as a single shoot. Weekly measurements were taken for stem length, number of nodes, internode length, number of leaves, leaf length (from petiole attachment point to the tip of the leaf down the midvein), leaf width (from one side of the leaf to the other at the widest point perpendicular to the midvein), leaf lobe sinus lengths and angles, leaf surface area, tendril emergence, and berry development (berry occurrence and circumference). Stem elongation rate was calculated from repeated stem length measurements. Leaf surface area was obtained from photographs using ImageJ version 1.52 [121]. Leaf measurements were performed weekly on at least ten plants per line and repeated continuously over at least 6 months. All measurements were performed on similar nodes to ensure uniform developmental stages. Shoots were pruned when the plant height reached $1 \mathrm{~m}$, at which time, measurements were repeated as a new shoot emerged at the cane. Leaf length measurements were repeated over the course of 3 years. To phenotype roots, overexpression line propagates were transferred to $12 \mathrm{~L}$ hydroponic tubs containing an air-stone and $0.5 \mathrm{x}$ strength Cramer's complete nutrient solution when roots were $\sim 5 \mathrm{~cm}$. Propagates were placed in tight fitting lids and allowed to grow for 20 days under greenhouse conditions. Roots were imaged and analyzed with WinRHIZO every 5 days. Measurements included total root length, total root surface area, number of primary lateral roots, number of adventitious roots, and plant fresh weight. Two mature leaves at similar developmental stage from each transgenic line from three individual cloned plants were excised from mature plants and frozen in liquid nitrogen for RNA sequencing. Berry occurrence, number and circumference were photographed and quantified with ImageJ.

\section{Abiotic stress and hormone treatments}

Treatments consisted of control treatment that entailed irrigating plants daily with $100 \mathrm{~mL}$ complete nutrient solution under greenhouse conditions (control); a salinity 
treatment: that was irrigating plants daily with $100 \mathrm{~mL}$ complete nutrient solution with $100 \mathrm{mM} \mathrm{NaCl}$ and 20 $\mathrm{mM} \mathrm{CaCl} \mathrm{Cl}_{2}$ added; a cold treatment: that was growing plants in a $10{ }^{\circ} \mathrm{C}$ growth chamber with a light intensity $50 \mu \mathrm{E}$ and irrigating daily with $100 \mathrm{~mL} 10^{\circ} \mathrm{C}$ complete nutrient solution; a water deficit treatment: that was maintaining pots at a low $30 \%$ relative water content. Water deficit pots were dried down to $30 \%$ relative water content by withholding irrigation at which point they were maintained daily at $30 \%$ pot relative water content, for 1 and 2 weeks. Control plants were watered in excess daily. After 20 days of salt, cold and control treatment, four experimental replicates of individual G1, L12-1, L12-2, L12-3, L12-11, and L12-23 vines were harvested. After 1 and 2 weeks of control and water deficit treatment, five experimental replicates of G1, L12-1, L12-2, L12-3, L12-11, and L12-23 individual vines were harvested. Shoot, stem, leaf, and root fresh and dry weights were measured in addition to total canopy surface area measured from photographs with ImageJ.

To examine VviERF6L1 response to hormones, CS leaves were sprayed with $10 \mu \mathrm{M}$ ProTone (s-ABA) (Valent BioSciences LLC) or water (control) for $1 \mathrm{~h}$. All sprays contained $0.5 \%$ Tween 20 . For spray treatments, mature leaves were selected and sprayed to saturation (solution dripping from leaves) on both sides of the leaf [122]. All samples were frozen in liquid nitrogen for all treatments. These experiments were performed in triplicate with each round consisting of three individual leaves of similar developmental stage from separate plants per genotype per harvest-time. Different sprays were made for each round.

Chilling treatments were performed on CS, RA, RI, and SC plants placed in a $4{ }^{\circ} \mathrm{C}$ growth chamber with a light intensity of $\sim 200 \mu \mathrm{mol} \mathrm{m}{ }^{-2} \mathrm{~s}^{-1}$ in a randomizedblock experimental design. Eight thermometers were placed evenly throughout pots in the growth chamber. Pot temperature was recorded before each harvest time. Control plants were kept under greenhouse conditions. Total leaves were harvested within 2 min after $2 \mathrm{~h}$ of chilling treatment and frozen in liquid nitrogen. These experiments were performed in triplicate with each round consisting of three individual plants per genotype per harvest-time per treatment. Additional chilling treatments were performed in a $4{ }^{\circ} \mathrm{C}$ refrigerator. $\mathrm{RA}$ and $\mathrm{CS}$ leaves of comparable age and size were placed on a wire support in pre-chilled or control Tupperware boxes containing $200 \mathrm{~mL}$ DI water similar to what has been described previously [123]. Petioles were placed in the water and lamina was supported by the wire rack above the water. A light-proof cardboard box was placed over the leaf-containing Tupperware box to prevent light intrusion. Control samples were placed under a light-proof box at $23^{\circ} \mathrm{C}$. Leaves were harvested within 2 min after 2 $h$ of temperature treatment and frozen in liquid nitrogen.

\section{RNA extraction}

All samples were ground with a mortar and pestle under liquid nitrogen. RNA extraction for RNA-Seq samples was performed with a CTAB-based method including an RNase-free DNase treatment as previously described [124]. RNA-Seq samples were prepared from $1.3 \mu \mathrm{g}$ RNA. Quality and concentration were confirmed with Ribogreen technology performed by the Nevada Genomics Center and Experion RNA StdSens Chips (Bio-Rad).

RNA from leaves of the plants treated with either abiotic or hormone treatment was extracted with a Spectrum Total Plant RNA kit (Sigma-Aldrich) modified protocol [125]. All RNA extractions were treated with RNAse-free DNase I (Qiagen) to remove genomic DNA contamination. RNA concentration, quantity, and purity for all samples was confirmed with a Nanodrop spectrophotometer, a $1.2 \%$ quality gel loaded with 400 ng RNA from each sample, and a $2 \%$ gel loaded with $10 \mu \mathrm{L}$ LAR intron PCR product. LAR PCR products were amplified from a $10 \mu \mathrm{L}$ GoTaq Green Master Mix (Promega) containing $250 \mathrm{ng}$ RNA and $0.5 \mu \mathrm{M}$ forward and reverse primers specific for a LAR intron. The PCR reaction included $95^{\circ} \mathrm{C}$ for $2 \mathrm{~min}, 35$ cycles of $95^{\circ} \mathrm{C}$ for $30 \mathrm{~s}, 62^{\circ} \mathrm{C}$ for $25 \mathrm{~s}$ and $72{ }^{\circ} \mathrm{C}$ for $25 \mathrm{~s}$. Purified samples demonstrating two bands corresponding to ribosomal subunit RNA, no band corresponding to the LAR intron, and sufficient concentration were utilized for RT-qPCR.

\section{PCR and RT-qPCR}

All samples from cold and hormone spray experiments were reverse transcribed with iScript Reverse Transcriptase Supermix (Bio-Rad) according to manufacturer's instructions from $2 \mu \mathrm{g}$ RNA. Primers were designed using NCBI Primer-BLAST. Primer sequences are provided in Additional File 38. Primer efficiencies were verified on purified PCR products (Machery-Nagel NucleoSpin ${ }^{\oplus}$ Gel and PCR Clean-up kit) and will be considered at $100 \%$ for the gene expression calculations. All reactions were performed on a Bio-Rad Real-Time thermal cycler CFX96 with the following protocol: $95^{\circ} \mathrm{C}$ for 3 mins; 40 cycles of $95^{\circ} \mathrm{C}$ for $10 \mathrm{~s}, 60^{\circ} \mathrm{C}$ for $15 \mathrm{~s}$. Fluorescence was recorded after each cycle, and melting curve analysis was performed from $65^{\circ} \mathrm{C}$ to $95^{\circ} \mathrm{C}$. Reference genes were selected based on a low coefficient of variation of expression reported in literature and uniform expression for all cDNA samples for each of the above described experiments.

VviERF6L1 overexpression was tested upon receival of transgenic plants with PCR of GFP and semiquantitative PCR of VviERF6L1. GFP expression was confirmed with PCR $\left(95^{\circ} \mathrm{C}\right.$ for 2 min, 35 cycles of $95^{\circ} \mathrm{C}$ 
for $30 \mathrm{~s}, 58^{\circ} \mathrm{C}$ for $30 \mathrm{~s}, 72^{\circ} \mathrm{C}$ for $30 \mathrm{~s}$ ) using GFP specific primers. Semi-quantitative PCR was performed on $1.0 \mu \mathrm{g}$ of reverse transcribed RNA from two leaves of three individual plants per line with gene specific primers. The reaction consisted of $7 \mu \mathrm{L} 10$-fold diluted cDNA, $3.5 \mu \mathrm{L}$ each $10 \mu \mathrm{M}$ forward and reverse primers, $35 \mu \mathrm{L}$ GoTaq Green Master Mix (Promega), and $21 \mu \mathrm{L}$ DEPC water. Samples collected at cycles 23, 26, 29, 32, and 35 were run on $2 \%$ agarose gels stained with ethidium bromide to compare VviERF6L1 amplification in overexpression lines relative to the empty vector control normalized to a ubiquitin reference gene. Several years after receival of the transgenic lines, stable overexpression was confirmed by RT-qPCR performed on $1.5 \mu \mathrm{g}$ cDNA from three individual leaves harvested on 3 separate plants for each line with gene specific primers as before [122]. RT-qPCR was performed on cDNA samples reverse transcribed form $1.3 \mu \mathrm{g}$ template RNA with iScript reverse transcriptase supermix (Bio-Rad) according to the manufacturer's instructions using VviGAPDH and VviACT7 as reference genes. RT-qPCR was conducted with SYBR Green Master Mix (Bio-Rad) for initial confirmation of overexpression lines and to confirm results on the same samples analyzed with RNA-Seq. All other RT-qPCR were performed with $2 \mu \mathrm{g}$ of RNA reverse transcribed to cDNA with iScript Reverse Transcriptase Supermix (Bio-Rad) according to manufacturer's instructions. RTqPCR was conducted for overexpression confirmation, chilling, $\mathrm{ABA}$, and respective control treatments with SYBR Green Master Mix (Bio-Rad). RT-qPCR was conducted to confirm VviERF6L1 expression and for cold treatments and controls with GoTaq ${ }^{\circ}$ qPCR Master Mix (Promega). Normalized relative quantity was calculated according to Hellemans et al. [101]. VviACT7 and VviGAPDH were used as reference genes for the ABA treatment. VviABF2 was used as a positive control gene for the ABA treatment. VviUbi and VviACT7 were used as reference genes, and VviCBF1 was used as a positive control gene.

\section{RNA-Seq analysis of VviERF6L1 overexpression lines}

Leaf RNA samples from three individual L12-3, L12-11, L12-23 and G1 vines were sequenced with Illumina TruSeq 2500 at the University of California, Los Angeles Genomic Center, to produce $36 \mathrm{bp}$ single end reads. Each sample was sequenced on two different lanes (technical replicates). Read quality for each sample was verified with FastQC version 0.11 .8 before and after trimming adaptors based on released adaptor sequences [117]. Sample 12-23-2_S8 had $\sim 4.4 \%$ library size compared to the average library size of the other samples. Sample 12-23-2_S8 was excluded from further analysis. Over-represented sequences were extracted and identified with blast+ version 2.8.0 alpha [126]. Reads from both sequencing lanes were concatenated per sample. Transcript abundance was quantified with Salmon with standard settings for single end reads [119]. Tximport version 1.8.0 [127] followed by DESeq2 version 1.20.0 [120] were implemented to perform differential expression analysis. Venn diagrams were created in $\mathrm{R}$ with the package limma version 3.36 .5 [117]. Heatmaps were created in R with ComplexHeatmap [128].

\section{Statistical analysis}

Statistical analysis performed to compare multiple means included the student t-test, one- and two-way ANOVAs followed by Tukey's HSD test after assumptions were met. Letters or asterisks indicate statistical significance between the specified comparisons. The error rate $\alpha=0.05$ was used in all comparisons. Statistical analyses were performed using $\mathrm{R}$ version 3.4.3.

\section{Supplementary information}

Supplementary information accompanies this paper at https://doi.org/10 1186/s12864-020-06811-8.

Additional file 1. PN40024 VviERF6L protein motif logos. Protein motif logos of PN40024 VviERF6Ls determined by MEME. X-axis represents relative residue position in motif. $Y$-axis letter height (bits) indicates relative frequency of a residue at a given position in the motif across the VviERF6L proteins. Left side colors correspond to Fig. 1.

Additional file 2. PN40024 and Cabernet Sauvignon (CS) VviERF6L protein motif consensus sequences. Motif number (given based on Evalue ranking), amino acid sequence, conservation (E-value), and length (amino acid residues) of the nine highly conserved protein motifs in PN40024 and CS VviERF6L proteins.

Additional file 3. PN40024 VviERF6L protein motif coordinates including consensus motif, and VviERF6L specific motif sequence, start and stop residue positions.

Additional file 4. PN40024 VviERF6L ERF domain percent identity with closest Arabidopsis thaliana ERF domain ortholog.

Additional file 5. The number of ERF6L paralogs across species. The number of ERF6Ls in species (carrot (D. carota), soybean (G. max), tomato (S. lycopersicum), and potato (S. tuberosum)) identified being closely related to VViERF6L1 from the Pantaxonomic Compara Gene Tree on Gramene (2018 version containing 44 species) using the V3 annotation of PN40024.

Additional file 6. Cabernet Sauvignon (CS) VviERF6L protein motif presence and abundance. The frequency of the 13 highly conserved amino acid motifs (right) in the 26 translated CS VviERF6L genes (bottom) Exact motif coordinates are in Additional File 8.

Additional file 7. Cabernet Sauvignon (CS) VviERF6L protein motif logos. Protein motif logos of CS VviERF6Ls determined by MEME. X-axis represents relative residue position in motif. Y-axis letter height (bits) indicates relative frequency of a residue at a given position in the motif across the VviERF6L proteins. Left side colors corresponding to PN40024 motifs based on percent identity.

Additional file 8. Cabernet Sauvignon (CS) VViERF6L protein motif coordinates including start position, motif number and consensus motif.

Additional file 9. CS VViERF6L protein motifs. Relative position of protein motifs from N-terminus (bottom) to C-terminus (top) of CS VviERF6Ls determined by MEME. Motif number based on E-value and sequence from Additional File 2 represented by colors (upper right). For exact motif coordinates of each CS VviERF6L see Additional File 8. CS VviERF6L motifs with corresponding PN40024 motifs share colors with Fig. 1. 
Additional file 10. Percent identity of PN40024 and Cabernet Sauvignon (CS) VviERF6L protein motifs.

Additional file 11. Cabernet Sauvignon (CS) VViERF6L gene names, protein sequences and protein length. Average length of all 26 at bottom.

Additional file 12. Maximum likelihood phylogenetic tree of PN40024 V2 assembly V3 structural annotation, $\mathrm{CS}, \mathrm{CH}$, and CA VviERF6L and al PN40024 VviERF proteins. Maximum likelihood method and Jones-TaylorThornton matrix-based model; Bootstrap consensus tree inferred from $n=$ 1,000 replicates. The percentage of bootstrap replicates in which associated proteins clustered together are shown as numbers next to the branches. The initial tree for the heuristic search generated from the maximum parsimony method. $n=217$ amino acid sequences and 1886 positions in the final data set. Evolutionary analysis from MEGA X; SubtreePruning-Regrafting - Fast (SPR level 3).

Additional file 13. PN40024, CS, CA, and CH VviERF6Ls and all PN40024 VviERF protein sequences used to create phylogenetic tree.

Additional file 14. PN40224 VviERF6L putative promoter region (-3000 bp) motif coordinates. Motif name, start site relative to transcription start site, sequence, PLACE identification number, Vitis gene name, and corresponding VViERF6L name are given.

Additional file 15. PN40024 VviERF6L promoter (-3000 bp) motif frequency. Including motif name, frequency in each VViERF6L denoted by common and gene names.

Additional file 16. Number and location of PN40024 cis-regulatory elements most abundant in VviERF6L12 relative to all other VViERF6L promoter regions. The ACGTATERD1 (red), LECPLEACS2 (green), SEF1MOTIF (blue), and WBOXATNPR1 (purple) were amongst the most abundant promoter motifs in VviERF6L12. Each occurrence of a motif is marked as a single hit at its appropriate position from the transcription start site (TSS) at position 0 . Motif nucleotide sequence denoted in corresponding color to hits. Complete cisregulatory element data is located in Additional Files 14 and 15.

Additional file 17. Expression of V ViERF6Ls across grapevine tissues and organs from the grapevine expression Atlas.

Additional file 18. VviERF6L gene expression in berry pulp, seed, and skin across berry development. Log2(RMA-normalized signal intensity+1) gene expression of 12 VviERF6Ls from Pinot Noir clone Pommard berry pulp (dark), seed (light), and skin (white) at pre-veraison (PRV), pink-soft (PS) berries at mid-ripening, and red-soft (RSH) berries at maturity [GSE49569]; mean \pm SE.

Additional file 19. Meta-data analysis of microarrays and RNA-seq series downloaded from NCBI GEO and SRA and investigated for VviERF6L expression

Additional file 20. Annotation and number of cross hybridizing VViERF6L probes.

Additional file 21. $R T-q P C R$ results of exogenous ABA application. Mature detached CS leaves were sprayed with exogenous 10 MM ABA (protone) or water control. Leaves were collected one hour after treatment. Control in pink and ABA treated in blue with bars as mean $\pm \mathrm{SE} ; n=3$.

Additional file 22. Differential expression analysis contrasts of interest for PRJNA516950. Including contrast number (arbitrarily assigned), species, treatment, organ, and week for both sample groups being compared.

Additional file 23. The number of differential expression analysis (DEA) contrasts of interest (COI) in which a VviERF6L was a differentially expressed gene (DEG) in PRJNA516950. VviERF6Ls are ordered from highest to lowest number of $\mathrm{COI}$ in which a VviERF6L is a DEG. COI are listed in Additional File 22

Additional file 24. VViERF6L gene expression in response to chilling. MAS5-calculated signal intensity of VviERF6Ls in CS shoot tips of vines exposed to $22^{\circ} \mathrm{C}$ control or $5^{\circ} \mathrm{C}$ chilling treatment for 0,4 , and 8 hours [GSE31594]; mean \pm SE.

Additional file 25. VViERF6L 1 did not respond to cold in Cabernet Sauvignon leaves. Expression of CBF1 (top) and VviERF6L1 (bottom) in CS leaves after 2 hours of $4^{\circ} \mathrm{C}$ chilling treatment represented as NRQ measured with $\mathrm{RT}$-qPCR, mean $\pm \mathrm{SE}, n=5$ rounds of three individual leaves from individual plants. Control and chilling are represented as blue and pink respectively.

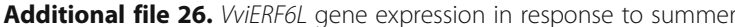
and winter harvest. $\log _{2}(\mathrm{FPKM}+1)$ gene expression of 12 V ViERF6Ls from berry pericarp of CS (light) and Riesling (dark) at three stages of ripening (EL35, 36, and 38) under a dual cropping system with harvesting in summer and winter [GSE103226]; mean \pm SE.

Additional file 27. VviERF6L gene expression in response to Erysiphe necator infection. $\log _{2}(T P M+1)$ gene expression of 18 V viERF6Ls from leaves of Vitis vinifera cv. Carignan and Chinese Vitis accession DVIT3351.27 (DVIT3351), Husseine, Karadshandal, Khalchii, O34-16, Sochal, and Valilov mock (dark) or inoculated (light) with Erysiphe necator 1- and 5-days post infection (DPI) [GSE67191]; mean \pm SE.

Additional file 28. VviERF6L gene expression in CS and SG pericarp over berry development across vineyards and years. $\log _{2}(\mathrm{RMA}$-normalized signal intensity+1) gene expression of 12 VviERF6Ls from Cabernet Sauvignon (CS (dark)) and Sangiovese (SG (light)) berry pericarp from three vineyards located in Bolgheri, Montalcino, and Riccone Italy in 2011 and 2012 over pea-size (PS), pre-veraison (PV), mid-ripening (MR), and fully ripened (FR) stages of development; mean \pm SE.

Additional file 29. VViERF6L gene expression in Corvina pericarp over berry development across vineyards and years. $\log _{2}(R M A-n o r m a l i z e d$ signal intensity+1) gene expression of 12 VviERF6Ls from Corvina pericarp from four representative Italian vineyards (abbreviated names from original paper; meaning nondisclosed) from 2006-2008 at veraison (V), mid-ripening (MR), and harvest (H) [GSE41633]; mean \pm SE.

Additional file 30. Venn diagram of gene co-expression analysis. Coexpression analysis was performed on the 18-member VviERF6L clade in the five data series reanalyzed with the PN40024 V3 annotation. Number of genes sharing expression patterns for data series represented in crosssections from top 100 co-expressed genes. Number at bottom indicates genes that did not share expression pattern with the VViERF6L clade.

Additional file 31. List of genes co-expressed with VviERF6L clade in four out of five data series. Gene ID and corresponding annotation. The four RNA-Seq data series the genes were co-expressed with the VViERF6L clade is listed.

Additional file 32. Fold increase of VViERF6L12 expression relative to average expression of all other VviERF6Ls. Expression value of VviERF6L12 and average value of all other VviERF6Ls across all conditions for each data series with each data series' respective units. Fold increase of V ViERF6L12 expression taken as ratio of VViERF6L12 expression relative to average expression value of all other VviERF6Ls across all conditions per data series.

Additional file 33. Verification of VviERF6L 1 overexpression lines. (A) Semi-quant RT-qPCR of GFP, VviERF6L1, and VviUbi1 from leaves at cycle 32. (B) Verification of VviERF6L1 overexpression with RT-qPCR with VviGAPDH and $V$ viACT7 reference genes from individual leaves represented as a normalized relative quantity, mean $\pm \mathrm{SE}, \mathrm{n}=3$ individual leaves from 3 individual plants. Stars indicate significance between G1 (empty vector control) and VviERF6L 1 overexpression lines ( $p$-value $<0.05$ ) using student's T-test. Blue and green corresponding to VviERF6L1 overexpression lines and empty vector control, respectively.

Additional file 34. Morphological phenotyping measurements taken that were not statistically significant between OX VViERF6L1 and empty vector control lines. Measurements were collected over the span of three weeks to 3 years. Data were tested for significant differences between empty vector control line (G1) and overexpression lines (L12-1, 2, 3, 11, and 23). Assumptions were met for tests used to determine significance.

Additional file 35. VviERF6L expression in VViERF6L 1 overexpression lines. For each overexpression line (L12-3, L12-11, L12-23) and the empty vector control (G1), an average TMP value was calculated and $\log _{2}$ transformed and colored from yellow (low value) to purple (high value) for each of the 18 VViERF $6 L s, n=3$

Additional file 36. Annotations of differentially expressed genes in three VViERF6L1 overexpression lines relative to empty vector control. 
Additional file 37. Venn Diagram of differentially expressed genes between $L 12-3,-11$, and -23 VviERF6L1 overexpression lines relative to G1 empty vector control. Number of upregulated genes presented in black and down regulated genes presented as grey.

Additional file 38. Primers used for RT-qPCR analysis. Primers were designed using primer 3 and NCBI's primer design tool. Primers were designed to be specific for genes of interest and respectful of RT-qPCR settings and specifications.

\section{Abbreviations}

\% ID: Percent identity; A + F: Acclimation with freezing; AA: Amino acid; ACC: Chilling acclimation; ACT7: Actin 7; At: Arabidopsis thaliana; bp: Base pair; C: Control; CA: Vitis vinifera (L.) cv. Carménère; CBF1: C-repeat/DRE binding factor 1; CDS: Coding sequence; CS: Vitis vinifera (L.) Cv. Cabernet Sauvignon clone 8; CH: Vitis vinifera (L.) Cv. Chardonnay; COl: Contrasts of interest; DEA: Differential expression analysis; DEG: Differentially expressed gene; DEPC: Diethyl pyrocarbonate; DI: De-ionized; ERF: Ethylene Response Factor; fdr: False discovery rate; FR: Fully ripened; FRZ: Freezing; GAPDH: Glyceraldehyde-3-Phosphate Dehydrogenase of Plastid; GEO: NCBI Gene Expression Omnibus; GFP: Green fluorescent protein; GH: Green-hard; GS: Green-soft; GSH: Green-soft at harvest; H: Harvest; Harv: Harvest; IW: Inoculated-wounded; JA: Jasmonic acid; LAR: Leucoanthocyanidin reductase; MEME: Multiple Em for Motif Elicitation; min: Minute; MR: Midripening; NCBI: National Center for Biotechnology Information; NINW: Noninoculated - non-wounded; NIW: Non-inoculated - wounded; NRQ: Normalized relative quantity; OX: Overexpression; Pea: The stage of berry development in which berries are the size of peas; Pro: 3000 base pairs from transcription start site; PRV: Pre-veraison; PS: Pea-sized; PSH: Pink-soft at harvest; PV: Post-veraison; RA: Vitis champinii (Ramsey); Rl: Vitis riparia (Riparia); RNA-Seq: Ribo-nucleic acid-sequencing; RS: Red-soft; RSH: Red-soft at harvest; RT-qPCR: Reverse transcription-quantitative polymerase chain reaction; s: Second; SC: Vitis vinifera $\times$ Vitis girdiana (SC2); SG: Sangiovese; Soft: The stage of berry development in which berries begin to soften; SRA: NCBI Sequence Read Archive; Touch: The stage of berry development in which berries begin to touch each other; TPM: Transcripts per million; TSS: Transcription start site; Vvilubi1: Vitis vinifera ubiquitin 1: W: Week: WD: Water deficit; WT: Wild type; V: Veraison; Vs.: Versus; VviERF6L1: Vitis vinifera Ethylene Response Factor 6-Like 1

\section{Acknowledgements}

The authors thank all the undergraduate student members of the laboratory for their technical assistance. We would like to recognize and thank Stefan Czemmel for his preliminary analysis.

\section{Authors' contributions}

GRC identified VviERF6Ls manually. NC and HT identified VviERF6Ls with the motif detection strategy in MEME. DG established the VviERF6L1 overexpression lines. NC and HT performed meta-data analysis. NC performed the co-expression analysis. NC performed RNA-Seq analysis on PRJNA516950 and VviERF6L1 overexpression lines (PRJNA605564). NC and HT performed promoter analysis. HT designed and performed ABA, chilling, and VviERF6L1 overexpression line experiments and RT-qPCR. HT phenotyped VViERF6L1 overexpression lines. HT processed and analyzed the data and wrote the body of the manuscript. All authors reviewed, edited, and approved the final version of the manuscript.

\section{Funding}

This work was supported by the USDA National Institute of Food and Agriculture Hatch project 1015139, which funded the graduate assistantship for the lead author and supplies to conduct this research project.

\section{Availability of data and materials}

RNA-Seq data from the VviERF6L1 overexpression and empty vector control lines were deposited in the Sequence Read Archive (SRA) database with the accession number PRJNA605564.

\section{Ethics approval and consent to participate} Not applicable.

\section{Consent for publication}

Not applicable.

\section{Competing interests}

The authors declare they have no competing interests. Grant R. Cramer is a member of the editorial board (section editor) of this journal.

\section{Author details}

'Department of Biochemistry and Molecular Biology, University of Nevada, Reno, NV 89557, USA. ${ }^{2}$ Precision Bred LLC, 16676 Sparrow Hawk Lane, Sonora, CA 95370, USA.

Received: 10 February 2020 Accepted: 8 June 2020

Published online: 09 July 2020

\section{References}

1. Iqbal N, Khan NA, Ferrante A, Trivellini A, Francini A, Khan MIR. Ethylene role in plant growth, development and senescence: interaction with other Phytohormones. Front Plant Sci. 2017;8. https://doi.org/10.3389/fpls.2017. 00475.

2. Licausi F, Ohme-Takagi M, Perata P. APETALA2/ethylene responsive factor (AP2/ERF) transcription factors: mediators of stress responses and developmental programs. New Phytol. 2013;199:639-49.

3. Zhu Z, Shi J, Xu W, Li H, He M, Xu Y, et al. Three ERF transcription factors from Chinese wild grapevine Vitis pseudoreticulata participate in different biotic and abiotic stress-responsive pathways. J Plant Physiol. 2013;170:923-33.

4. Licausi F, Giorgi FM, Zenoni S, Osti F, Pezzotti M, Perata P. Genomic and transcriptomic analysis of the AP2/ERF superfamily in Vitis vinifera. BMC Genomics. 2010;11. https://doi.org/10.1186/1471-2164-11-719.

5. Ma Q, Zhang G, Hou L, Wang W, Hao J, Liu X. Vitis vinifera VvWRKY13 is an ethylene biosynthesis-related transcription factor. Plant Cell Rep. 2015;34:1593-603.

6. Chervin C, El-Kereamy A, Roustan J-P, Latché A, Lamon J, Bouzayen M. Ethylene seems required for the berry development and ripening in grape, a non-climacteric fruit. Plant Sci. 2004:167:1301-5.

7. Leida C, Dal Rì A, Dalla Costa L, Gómez MD, Pompili V, Sonego P, et al. Insights into the role of the berry-specific ethylene responsive factor VviERF045. Front Plant Sci. 2016;7. https://doi.org/10.3389/fpls.2016.01793.

8. Müller M, Munné-Bosch S. Ethylene response factors: a key regulatory hub in hormone and stress signaling. Plant Physiol. 2015;169:32-41.

9. Burg SP, Burg EA. The interaction between auxin and ethylene and its role in plant growth. PNAS. 1966:55:262-9.

10. Sharp RE. Interaction with ethylene: changing views on the role of abscisic acid in root and shoot growth responses to water stress. Plant Cell Environ. 2002;25:211-22.

11. Kunkel BN, Brooks DM. Cross talk between signaling pathways in pathogen defense. Curr Opin Plant Biol. 2002;5:325-31.

12. Saithong T, Saerue S, Kalapanulak S, Sojikul P, Narangajavana J, Bhumiratana S. Gene co-expression analysis inferring the crosstalk of ethylene and gibberellin in modulating the transcriptional acclimation of cassava root growth in different seasons. PLoS One. 2015;10. https://doi.org/10.1371/ journal.pone.0137602.

13. Deslauriers SD, Larsen PB. FERONIA is a key modulator of Brassinosteroid and ethylene responsiveness in Arabidopsis hypocotyls. Mol Plant. 2010;3:626-40.

14. Divi UK, Rahman T, Krishna P. Brassinosteroid-mediated stress tolerance in Arabidopsis shows interactions with abscisic acid, ethylene and salicylic acid pathways. BMC Plant Biol. 2010;10. https://doi.org/10.1186/ 1471-2229-10-151.

15. Nakano T, Suzuki K, Fujimura T, Shinshi H. Genome-wide analysis of the ERF gene family in Arabidopsis and Rice. Plant Physiol. 2006;140:411-32.

16. Cramer GR, Ghan R, Schlauch KA, Tillett RL, Heymann H, Ferrarini A, et al. Transcriptomic analysis of the late stages of grapevine (Vitis vinifera CV. Cabernet sauvignon) berry ripening reveals significant induction of ethylene signaling and flavor pathways in the skin. BMC Plant Biol. 2014;14. https:// doi.org/10.1186/s12870-014-0370-8.

17. Hopper DW, Ghan R, Schlauch KA, Cramer GR. Transcriptomic network analyses of leaf dehydration responses identify highly connected $A B A$ and ethylene signaling hubs in three grapevine species differing in drought tolerance. BMC Plant Biol. 2016;16. https:/doi.org/10.1186/s12870-016-0804-6. 
18. Canaguier A, Grimplet J, Di Gaspero G, Scalabrin S, Duchêne E, Choisne N, et al. A new version of the grapevine reference genome assembly (12X.v2) and of its annotation (VCost.v3). Genom Data. 2017;14:56-62.

19. Sterck L, Billiau K, Abeel T, Rouzé P, Van de Peer Y. ORCAE: online resource for community annotation of eukaryotes. Nat Methods. 2012;9:1041.

20. Jaillon O, Aury J-M, Noel B, Policriti A, Clepet C, Casagrande A, et al. The grapevine genome sequence suggests ancestral hexaploidization in major angiosperm phyla. Nature. 2007;449:463-7.

21. Grimplet J, Van Hemert J, Carbonell-Bejerano P, Díaz-Riquelme J, Dickerson J, Fennell A, et al. Comparative analysis of grapevine whole-genome gene predictions, functional annotation, categorization and integration of the predicted gene sequences. BMC Res Notes. 2012;5. https://doi.org/10.1186/ 1756-0500-5-213.

22. Bailey TL, Boden M, Buske FA, Frith M, Grant CE, Clementi L, et al. MEME Suite: tools for motif discovery and searching. Nucleic Acids Res. 2009; 37(Web Server issue):W202-8

23. Gupta S, Stamatoyannopoulos JA, Bailey TL, Noble WS. Quantifying similarity between motifs. Genome Biol. 2007:8:R24.1-9.

24. Waterhouse A, Bertoni M, Bienert S, Studer G, Tauriello G, Gumienny R, et al. SWISS-MODEL: homology modelling of protein structures and complexes. Nucleic Acids Res. 2018;46(Web server issue):W296-303.

25. Mitchell AL, Attwood TK, Babbitt PC, Blum M, Bork P, Bridge A, et al. InterPro in 2019: improving coverage, classification and access to protein sequence annotations. Nucleic Acids Res. 2019;47(Database issue):D351-60.

26. Tello-Ruiz MK, Naithani S, Stein JC, Gupta P, Campbell M, Olson A, et al. Gramene 2018: unifying comparative genomics and pathway resources for plant research. Nucleic Acids Res. 2018;46(Database issue):D1181-9.

27. Chin C-S, Peluso P, Sedlazeck FJ, Nattestad M, Concepcion GT, Clum A, et al. Phased diploid genome assembly with single-molecule real-time sequencing. Nat Methods. 2016;13:1050-4

28. Altschul SF, Gish W, Miller W, Myers EW, Lipman DJ. Basic local alignment search tool. J Mol Biol. 1990:215:403-10.

29. Zhou Y, Minio A, Massonnet M, Solares E, LV Y, Beridze T, et al. The population genetics of structural variants in grapevine domestication. Nat Plants. 2019;5:965-79.

30. Minio A, Massonnet M, Figueroa-Balderas R, Castro A, Cantu D. Diploid Genome Assembly of the Wine Grape Carménère. G3. 2019;9:1331-7.

31. Kumar S, Stecher G, Li M, Knyaz C, Tamura K. MEGA X: Molecular evolutionary genetics analysis across computing platforms. Mol Biol Evol. 2018;35:1547-9.

32. Higo K, Ugawa Y, Iwamoto M, Korenaga T. Plant cis-acting regulatory DNA elements (PLACE) database: 1999. Nucleic Acids Res. 1999:27:297-300.

33. Fasoli M, Dal Santo S, Zenoni S, Tornielli GB, Farina L, Zamboni A, et al. The grapevine expression atlas reveals a deep Transcriptome shift driving the entire plant into a maturation program. Plant Cell. 2012;24: 3489-505.

34. Gouthu S, O'Neil ST, Di Y, Ansarolia M, Megraw M, Deluc LG. A comparative study of ripening among berries of the grape cluster reveals an altered transcriptional programme and enhanced ripening rate in delayed berries. J Exp Bot. 2014;65:5889-902.

35. Edgar R. Gene expression omnibus: NCBI gene expression and hybridization array data repository. Nucleic Acids Res. 2002;30:207-10.

36. Leinonen R, Sugawara H, Shumway M. International Nucleotide Sequence Database Collaboration. The Sequence Read Archive. Nucleic Acids Research. 2011;39(Database):D19-21.

37. Cramer GR, Ergül A, Grimplet J, Tillett RL, Tattersall EAR, Bohlman MC, et al. Water and salinity stress in grapevines: early and late changes in transcript and metabolite profiles. Funct Integr Genomics. 2007;7:111-34.

38. Cochetel N, Ghan R, Toups HS, Degu A, Tillett RL, Schlauch KA, et al. Drought tolerance of the grapevine, Vitis champinii cv. Ramsey, is associated with higher photosynthesis and greater transcriptomic responsiveness of abscisic acid biosynthesis and signaling. BMC Plant Biol. 2020;20. https://doi.org/10.1186/s12870-019-2012-7.

39. Londo JP, Kovaleski AP, Lillis JA. Divergence in the transcriptional landscape between low temperature and freeze shock in cultivated grapevine (Vitis vinifera). Hortic Res. 2018;5. https://doi.org/10.1038/s41438-018-0020-7.

40. Tattersall EAR, Grimplet J, DeLuc L, Wheatley MD, Vincent D, Osborne C, et al. Transcript abundance profiles reveal larger and more complex responses of grapevine to chilling compared to osmotic and salinity stress. Funct Integr Genomics. 2007;7:317-33.
41. Sun R-Z, Cheng G, Li Q, He Y-N, Wang Y, Lan Y-B, et al. Light-induced Variation in Phenolic Compounds in Cabernet Sauvignon Grapes (Vitis vinifera L.) Involves Extensive Transcriptome Reprogramming of Biosynthetic Enzymes, Transcription Factors, and Phytohormonal Regulators. Front Plant Sci. 2017;8. https://doi.org/10.3389/fpls.2017.00547.

42. Chen W-K, Bai X-J, Cao M-M, Cheng G, Cao X-J, Guo R-R, et al. Dissecting the variations of ripening progression and flavonoid metabolism in grape berries grown under double cropping system. Front Plant Sci. 2017;8. https://doi.org/10.3389/fpls.2017.01912.

43. Massonnet M, Figueroa-Balderas R, Galarneau ERA, Miki S, Lawrence DP, Sun $Q$, et al. Neofusicoccum parvum colonization of the grapevine Woody stem triggers asynchronous host responses at the site of infection and in the leaves. Front Plant Sci. 2017:8. https://doi.org/10.3389/fpls.2017.01117.

44. Amrine KCH, Blanco-Ulate B, Riaz S, Pap D, Jones L, Figueroa-Balderas R, et al. Comparative transcriptomics of central Asian Vitis vinifera accessions reveals distinct defense strategies against powdery mildew. Hortic Res. 2015;2. https://doi.org/10.1038/hortres.2015.37.

45. Blanco-Ulate B, Hopfer H, Figueroa-Balderas R, Ye Z, Rivero RM, Albacete A, et al. Red blotch disease alters grape berry development and metabolism by interfering with the transcriptional and hormonal regulation of ripening. J Exp Bot. 2017;68:1225-38.

46. Massonnet M, Fasoli M, Tornielli GB, Altieri M, Sandri M, Zuccolotto P, et al. Ripening Transcriptomic program in red and white grapevine varieties correlates with berry skin anthocyanin accumulation. Plant Physiol. 2017;174: 2376-96.

47. Dal Santo S, Zenoni S, Sandri M, De Lorenzis G, Magris G, De Paoli E, et al. Grapevine field experiments reveal the contribution of genotype, the influence of environment and the effect of their interaction $(G \times E)$ on the berry transcriptome. Plant J. 2018;93:1143-59.

48. Dal Santo S, Tornielli GB, Zenoni S, Fasoli M, Farina L, Anesi A, et al. The plasticity of the grapevine berry transcriptome. Genome Biol. 2013;14. https://doi.org/10.1186/gb-2013-14-6-r54.

49. Gray D, Zhijian, Li. pECBC Patent. 2006. https://patentimages.storage. googleapis.com/de/c6/75/230d1ee352af94/US7129343.pdf.

50. Wang $L$, Sun $Y$, Xia $X-L$, Jiang T-B. Screening of proteins interacting with ERF transcriptional factor from Populus simonii $\times$ P.nigra by yeast two-hybrid method. Biotechnol Biotechnol Equip. 2018;32:543-9.

51. Alves M, Dadalto S, Gonçalves A, de Souza G, Barros V, Fietto L. Transcription factor functional protein-protein interactions in plant defense responses. Proteomes. 2014;2:85-106.

52. Phukan UJ, Jeena GS, Tripathi V, Shukla RK. Regulation of Apetala2/ethylene response factors in plants. Front Plant Sci. 2017;8. https://doi.org/10.3389/ fpls.2017.00150

53. Bethke G, Unthan T, Uhrig JF, Poschl Y, Gust AA, Scheel D, et al. Flg22 regulates the release of an ethylene response factor substrate from MAP kinase 6 in Arabidopsis thaliana via ethylene signaling. PNAS. 2009;106:8067-72.

54. Panchy N, Lehti-Shiu MD, Shiu S-H. Evolution of gene duplication in plants. Plant Physiol. 2016;171:2294-316.

55. Renny-Byfield S, Gallagher JP, Grover CE, Szadkowski E, Page JT, Udall JA et al. Ancient gene duplicates in Gossypium (cotton) exhibit near-complete expression divergence. GBE. 2014;6:559-71.

56. Brenchley R, Spannagl M, Pfeifer M, Barker GLA, D'Amore R, Allen AM, et al. Analysis of the bread wheat genome using whole-genome shotgun sequencing. Nature. 2012;491:705-10.

57. Schmutz J, Cannon SB, Schlueter J, Ma J, Mitros T, Nelson W, et al. Genome sequence of the palaeopolyploid soybean. Nature. 2010;463:178-83.

58. Schnable JC, Springer NM, Freeling M. Differentiation of the maize subgenomes by genome dominance and both ancient and ongoing gene loss. PNAS. 2011;108:4069-74

59. Wang X, Lin S, Liu D, Wang Q, McAvoy R, Ding J, et al. Characterization and expression analysis of ERF genes in Fragaria vesca suggest different divergences of tandem ERF duplicates. Front Genet. 2019;10. https://doi.org/ 10.3389/fgene.2019.00805.

60. Li X, Tao S, Wei S, Ming M, Huang X, Zhang S, et al. The mining and evolutionary investigation of AP2/ERF genes in pear (Pyrus). BMC Plant Biol. 2018;18. https://doi.org/10.1186/s12870-018-1265-x.

61. Shirsat A, Wilford N, Croy R, Boulter D. Sequences responsible for the tissue specific promoter activity of a pea legumin gene in tobacco. Mol Gen Genet. 1989;215:326-31.

62. Alves GSC, Torres LF, Déchamp E, Breitler J-C, Joët T, Gatineau F, et al. Differential fine-tuning of gene expression regulation in coffee leaves by 
CCDREB1D promoter haplotypes under water deficit. J Exp Bot. 2017;68: 3017-31.

63. Yanagisawa S. The Dof family of plant transcription factors. Trends Plant Sci. 2002;7:555-60

64. Giraud E, Ho LHM, Clifton R, Carroll A, Estavillo G, Tan Y-F, et al. The absence of ALTERNATIVE OXIDASE1a in Arabidopsis results in acute sensitivity to combined light and drought stress. Plant Physiol. 2008;147:595-610.

65. Kagale S, Links MG, Rozwadowski K. Genome-wide analysis of ethyleneresponsive element binding factor-associated Amphiphilic repression motifcontaining transcriptional regulators in Arabidopsis. Plant Physiol. 2010;152: 1109-34.

66. McGrath KC. Repressor- and activator-type ethylene response factors functioning in Jasmonate signaling and disease resistance identified via a genome-wide screen of Arabidopsis transcription factor gene expression. Plant Physiol. 2005;139:949-59.

67. Song C-P. Role of an Arabidopsis AP2/EREBP-type transcriptional repressor in Abscisic acid and drought stress responses. Plant Cell. 2005;17:2384-96.

68. Yang Z, Tian L, Latoszek-Green M, Brown D, Wu K. Arabidopsis ERF4 is a transcriptional repressor capable of modulating ethylene and abscisic acid responses. Plant Mol Biol. 2005;58:585-96.

69. Ohta M, Matsui K, Hiratsu K, Shinshi H, Ohme-Takagi M. Repression domains of class II ERF transcriptional repressors share an essential motif for active repression. Plant Cell. 2001;13:1959-68.

70. Yoshida T, Mogami J, Yamaguchi-Shinozaki K. ABA-dependent and ABAindependent signaling in response to osmotic stress in plants. Curr Opin Plant Biol. 2014;21:133-9.

71. Wang L, Waters MT, Smith SM. Karrikin-KAI2 signalling provides Arabidopsis seeds with tolerance to abiotic stress and inhibits germination under conditions unfavourable to seedling establishment. New Phytol. 2018;219:605-18.

72. Minio A, Massonnet M, Figueroa-Balderas R, Vondras AM, Blanco-Ulate B, Cantu D. Iso-Seq allows genome-independent Transcriptome profiling of grape berry Development. G3 (Bethesda). 2019;9:755-67.

73. Ali S, Mir ZA, Bhat JA, Tyagi A, Chandrashekar N, Yadav P, et al. Isolation and characterization of systemic acquired resistance marker gene PR1 and its promoter from Brassica juncea. 3 Biotech. 2018;8. https://doi.org/10.1007/ s13205-017-1027-8.

74. Bowling SA, Clarke JD, Liu Y, Klessig DF, Dong X. The cpr5 mutant of Arabidopsis expresses both NPRI-dependent and NPRI-1ndependent resistance. Plant Cell. 1997;9:1573-84.

75. Ford KA, Casida JE, Chandran D, Gulevich AG, Okrent RA, Durkin KA, et al. Neonicotinoid insecticides induce salicylate-associated plant defense responses. PNAS. 2010;107:17527-32.

76. Laird J, Armengaud P, Giuntini P, Laval V, Milner JJ. Inappropriate annotation of a key defence marker in Arabidopsis: will the real PR-1 please stand up? Planta. 2004:219:1089-92.

77. Moffat CS, Ingle RA, Wathugala DL, Saunders NJ, Knight H, Knight MR. ERF5 and ERF6 play redundant roles as positive regulators of JA/et-mediated defense against Botrytis cinerea in Arabidopsis. PLoS One. 2012;7. https:// doi.org/10.1371/journal.pone.0035995.

78. Berthet S, Thevenin J, Baratiny D, Demont-Caulet N, Debeaujon I, Bidzinski P, et al. Role of plant Laccases in lignin polymerization. In: Advances in Botanical Research. Elsevier; 2012. p. 145-72. https://doi.org/10.1016/B978-012-416023-1.00005-7

79. Lee B-R, Kim K-Y, Jung W-J, Avice J-C, Ourry A, Kim T-H. Peroxidases and lignification in relation to the intensity of water-deficit stress in white clover (Trifolium repens L.). J Exp Bot. 2007;58:1271-9.

80. Fan L, Linker R, Gepstein S, Tanimoto E, Yamamoto R, Neumann PM. Progressive inhibition by water deficit of Cell Wall extensibility and growth along the elongation zone of maize roots is related to increased lignin metabolism and progressive Stelar accumulation of wall Phenolics. Plant Physiol. 2006;140:603-12.

81. Son GH, Wan J, Kim HJ, Nguyen XC, Chung WS, Hong JC, et al. Ethyleneresponsive element-binding factor 5, ERF5, is involved in chitin-induced innate immunity response. MPMI. 2012;25:48-60.

82. Warmerdam S, Sterken MG, Van Schaik C, Oortwijn MEP, Lozano-Torres JL, Bakker J, et al. Mediator of tolerance to abiotic stress ERF6 regulates susceptibility of Arabidopsis to Meloidogyne incognita. Mol Plant Pathol. 2018;20:137-52.

83. Fröschel C, Iven T, Walper E, Bachmann V, Weiste C, Dröge-Laser W. A gain-of-function screen reveals redundant ERF transcription factors providing opportunities for resistance breeding toward the vascular fungal pathogen Verticillium longisporum. Mol Plant-Microbe Interact. 2019;32:1095-109.

84. Rienth M, Crovadore J, Ghaffari S, Lefort F. Oregano essential oil vapour prevents Plasmopara viticola infection in grapevine (Vitis Vinifera) and primes plant immunity mechanisms. PLoS One. 2019;14. https://doi.org/10. 1371/journal.pone.0222854.

85. Eisenmann B, Czemmel S, Ziegler T, Buchholz G, Kortekamp A, Trapp O, et al. Rpv3-1 mediated resistance to grapevine downy mildew is associated with specific host transcriptional responses and the accumulation of stilbenes. BMC Plant Biol. 2019;19. https://doi.org/10. 1186/s12870-019-1935-3.

86. Liu M, Gomes BL, Mila I, Purgatto E, Peres LEP, Frasse P, et al. Comprehensive profiling of ethylene response factor expression identifies ripening-associated ERF genes and their link to key regulators of fruit ripening in tomato. Plant Physiol. 2016;170:1732-44.

87. Fernandez-Pozo N, Zheng Y, Snyder SI, Nicolas P, Shinozaki Y, Fei Z, et al. The tomato expression atlas. Bioinformatics. 2017;33:2397-8.

88. Liu M, Diretto G, Pirrello J, Roustan J-P, Li Z, Giuliano G, et al. The chimeric repressor version of an ethylene response factor (ERF) family member, SIERF.B3, shows contrasting effects on tomato fruit ripening. New Phytol. 2014:203:206-18.

89. Benfey PN, Linstead PJ, Roberts K, Schiefelbein JW, Hauser MT, Aeschbacher RA. Root development in Arabidopsis: four mutants with dramatically altered root morphogenesis. Development. 1993;119:57-70.

90. Pysh LD, Wysocka-Diller JW, Camilleri C, Bouchez D, Benfey PN. The GRAS gene family in Arabidopsis: sequence characterization and basic expression analysis of the SCARECROW-LIKE genes. Plant J. 1999;18:111-9.

91. Grimplet J, Agudelo-Romero P, Teixeira RT, Martinez-Zapater JM, Fortes AM. Structural and functional analysis of the GRAS gene family in grapevine indicates a role of GRAS proteins in the control of development and stress responses. Front Plant Sci. 2016;7. https://doi.org/10.3389/fpls.2016.00353.

92. Huang W, Xian Z, Kang X, Tang N, Li Z. Genome-wide identification, phylogeny and expression analysis of GRAS gene family in tomato. BMC Plant Biol. 2015;15:209.

93. Zhu Y, Dong A, Meyer D, Pichon O, Renou J-P, Cao K, et al. Arabidopsis NRP1 and NRP2 encode histone chaperones and are required for maintaining postembryonic root growth. Plant Cell. 2006;18:2879-92.

94. Sotelo-Silveira M, Cucinotta M, Chauvin A-L, Chavez Montes RA, Colombo L, Marsch-Martinez N, et al. Cytochrome P450 CYP78A9 is involved in Arabidopsis reproductive development. Plant Physiol. 2013;162:779-99.

95. Skirycz A, Claeys H, De Bodt S, Oikawa A, Shinoda S, Andriankaja M, et al. Pause-and-stop: the effects of osmotic stress on cell proliferation during early leaf development in Arabidopsis and a role for ethylene signaling in cell cycle arrest. Plant Cell. 2011;23:1876-88.

96. Dubois M, Skirycz A, Claeys H, Maleux K, Dhondt S, De Bodt S, et al. ETHYLENE RESPONSE FACTOR6 acts as a central regulator of leaf growth under water-limiting conditions in Arabidopsis. Plant Physiol. 2013;162:319-32.

97. Mase K, Ishihama N, Mori H, Takahashi H, Kaminaka H, Kodama M, et al Ethylene-responsive AP2/ERF transcription factor MACD1 participates in Phytotoxin-triggered programmed cell death. MPMI. 2013;26:868-79.

98. Sewelam N, Kazan K, Thomas-Hall SR, Kidd BN, Manners JM, Schenk PM. Ethylene response factor 6 is a regulator of reactive oxygen species signaling in Arabidopsis. PLoS One. 2013;8. https://doi.org/10.1371/journal.pone.0070289.

99. Dubois M, Van den Broeck L, Claeys H, Van Vlierberghe K, Matsui M, Inzé D. The ETHYLENE RESPONSE FACTORs ERF6 and ERF11 antagonistically regulate Mannitol-induced growth inhibition in Arabidopsis. Plant Physiol. 2015;169:166-79.

100. Rieu I, Eriksson S, Powers SJ, Gong F, Griffiths J, Woolley L, et al. Genetic analysis reveals that C19-GA 2-oxidation is a major gibberellin inactivation pathway in Arabidopsis. Plant Cell. 2008;20:2420-36.

101. Birkenbihl RP, Diezel C, Somssich IE. Arabidopsis WRKY33 is a key transcriptional regulator of hormonal and metabolic responses toward Botrytis cinerea infection. Plant Physiol. 2012;159:266-85.

102. Gigolashvili T, Berger B, Mock H-P, Müller C, Weisshaar B, Flügge U-I. The transcription factor HIG1/MYB51 regulates indolic glucosinolate biosynthesis in Arabidopsis thaliana: HIG1 and glucosinolate biosynthesis. Plant J. 2007; 50:886-901.

103. Wang X, Du B, Liu M, Sun N, Qi X. Arabidopsis transcription factor WRKY33 is involved in drought by directly regulating the expression of CesA8. AJPS. $2013 ; 4: 21-7$ 
104. Li S, Fu Q, Chen L, Huang W, Yu D. Arabidopsis thaliana WRKY25, WRKY26, and WRKY33 coordinate induction of plant thermotolerance. Planta. 2011; 233:1237-52.

105. Sakamoto H, Maruyama K, Sakuma Y, Meshi T, Iwabuchi M, Shinozaki K, et al. Arabidopsis Cys2/His2-type zinc-finger proteins function as transcription repressors under drought, cold, and high-salinity stress conditions. Plant Physiol. 2004;136:2734-46.

106. Klay I, Gouia S, Liu M, Mila I, Khoudi H, Bernadac A, et al. Ethylene response factors (ERF) are differentially regulated by different abiotic stress types in tomato plants. Plant Sci. 2018;274:137-45.

107. Yamaguchi-Shinozaki K, Shinozaki K. Organization of cis-acting regulatory elements in osmotic- and cold-stress-responsive promoters. Trends Plant Sci. 2005;10:88-94.

108. van Loon LC, Geraats BPJ, Linthorst HJM. Ethylene as a modulator of disease resistance in plants. Trends Plant Sci. 2006:11:184-91.

109. Ecker JR, Davis RW. Plant defense genes are regulated by ethylene. PNAS. 1987;84:5202-6.

110. Cheng C-Y, Krishnakumar V, Chan AP, Thibaud-Nissen F, Schobel S, Town CD. Araport11: a complete reannotation of the Arabidopsis thaliana reference genome. Plant J. 2017;89:789-804.

111. Vitulo N, Forcato C, Carpinelli EC, Telatin A, Campagna D, D'Angelo M, et al. A deep survey of alternative splicing in grape reveals changes in the splicing machinery related to tissue, stress condition and genotype. BMC Plant Biol. 2014;14

112. Berardini TZ, Reiser L, Li D, Mezheritsky Y, Muller R, Strait E, et al. The arabidopsis information resource: making and mining the "gold standard" annotated reference plant genome: Tair: making and mining the "gold standard" plant genome. Genesis. 2015;53:474-85.

113. Bodenhofer U, Bonatesta E, Horejš-Kainrath C, Hochreiter S. msa: an R package for multiple sequence alignment. 2015. https://academic.oup.com/ bioinformatics/article-lookup/doi/10.1093/bioinformatics/btv494. Accessed 9 May 2019.

114. Bailey TL, Elkan C. Fitting a mixture model by expectation maximization to discover motifs in biopolymers. Proc Int Conf Intell Syst Mol Biol. 1994;2:28-36.

115. Lawrence M, Huber W, Pagès H, Aboyoun P, Carlson M, Gentleman R, et al. Software for computing and annotating genomic ranges. PLoS Comput Biol. 2013;9. https://doi.org/10.1371/journal.pcbi.1003118.

116. Davis S, Meltzer PS. GEOquery: a bridge between the gene expression omnibus (GEO) and BioConductor. Bioinformatics. 2007:23:1846-7.

117. Andrews S. FastQC A Quality Control tool for High Throughput Sequence Data; 2014.

118. Bolger AM, Lohse M, Usadel B. Trimmomatic: a flexible trimmer for Illumina sequence data. Bioinformatics. 2014;30:2114-20.

119. Patro R, Duggal G, Love MI, Irizarry RA, Kingsford C. Salmon provides fast and bias-aware quantification of transcript expression. Nat Methods. 2017;14:417-9

120. Love MI, Huber W, Anders S. Moderated estimation of fold change and dispersion for RNA-seq data with DESeq2. Genome Biol. 2014;15. https://doi. org/10.1186/s13059-014-0550-8

121. Schneider CA, Rasband WS, Eliceiri KW. NIH image to ImageJ: 25 years of image analysis. Nat Methods. 2012;9:671-5.

122. Rattanakon S, Ghan R, Gambetta GA, Deluc LG, Schlauch KA, Cramer GR. Abscisic acid transcriptomic signaling varies with grapevine organ. BMC Plant Biol. 2016;16. https://doi.org/10.1186/s12870-016-0763-y.

123. Hopper DW, Ghan R, Cramer GR. A rapid dehydration leaf assay reveals stomatal response differences in grapevine genotypes. Hortic Res. 2014;1. https://doi.org/10.1038/hortres.2014.2.

124. Ghan R, Petereit J, Tillett RL, Schlauch KA, Toubiana D, Fait A, et al. The common transcriptional subnetworks of the grape berry skin in the late stages of ripening. BMC Plant Biol. 2017;17. https://doi.org/10.1186/s12870017-1043-1.

125. Cookson SJ, Clemente Moreno MJ, Hevin C, Nyamba Mendome LZ, Delrot S, Trossat-Magnin C, et al. Graft union formation in grapevine induces transcriptional changes related to cell wall modification, wounding, hormone signalling, and secondary metabolism. J Exp Bot. 2013;64:2997-3008.

126. Camacho C, Coulouris G, Avagyan V, Ma N, Papadopoulos J, Bealer K, et al. BLAST+: architecture and applications. BMC Bioinformatics. 2009;10. https:// doi.org/10.1186/1471-2105-10-421.
127. Soneson C, Love MI, Robinson MD. Differential analyses for RNA-seq: transcript-level estimates improve gene-level inferences. F1000Res. 2015:4:1521

128. Gu Z, Eils R, Schlesner M. Complex heatmaps reveal patterns and correlations in multidimensional genomic data. Bioinformatics. 2016;32: 2847-9.

\section{Publisher's Note}

Springer Nature remains neutral with regard to jurisdictional claims in published maps and institutional affiliations.
Ready to submit your research? Choose BMC and benefit from:

- fast, convenient online submission

- thorough peer review by experienced researchers in your field

- rapid publication on acceptance

- support for research data, including large and complex data types

- gold Open Access which fosters wider collaboration and increased citations

- maximum visibility for your research: over $100 \mathrm{M}$ website views per year

At BMC, research is always in progress.

Learn more biomedcentral.com/submissions 